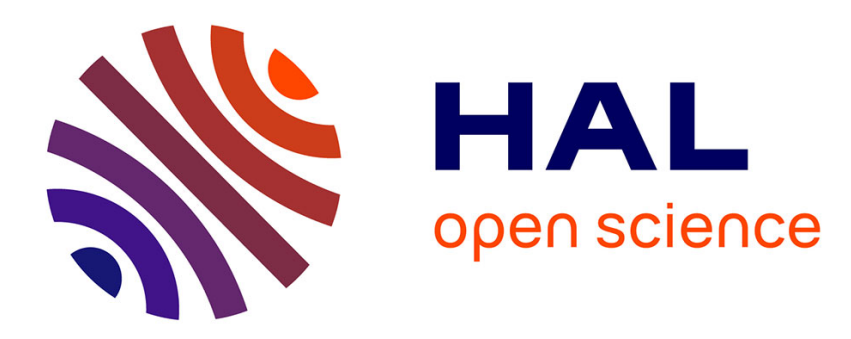

\title{
Stationary Solutions to Phase Field Crystal Equations
}

Morgan Pierre, Arnaud Rougirel

\section{To cite this version:}

Morgan Pierre, Arnaud Rougirel. Stationary Solutions to Phase Field Crystal Equations. 2009. hal-00407353

\section{HAL Id: hal-00407353 \\ https://hal.science/hal-00407353}

Preprint submitted on 24 Jul 2009

HAL is a multi-disciplinary open access archive for the deposit and dissemination of scientific research documents, whether they are published or not. The documents may come from teaching and research institutions in France or abroad, or from public or private research centers.
L'archive ouverte pluridisciplinaire HAL, est destinée au dépôt et à la diffusion de documents scientifiques de niveau recherche, publiés ou non, émanant des établissements d'enseignement et de recherche français ou étrangers, des laboratoires publics ou privés. 


\title{
STATIONARY SOLUTIONS TO PHASE FIELD CRYSTAL EQUATIONS
}

\author{
MORGAN PIERRE \& ARNAUD ROUGIREL
}

\begin{abstract}
This paper is devoted to the analytical and numerical study of the stationary solutions of the one dimensional Phase Field Crystal Equation. This new model recently introduced by K. Elder and M. Grant describes phase transformations at atomistic level on large time scales. By using bifurcation methods, we investigate quantitative and qualitative properties of these solutions: multiplicity, stability, periodicity. Quite unusual bifurcation diagrams are obtained by numerical simulations.
\end{abstract}

\section{INTRODUCTION}

In materials science, the study of phase transformations is a very challenging issue. Since material properties depend on microstructures that form during non equilibrium processing, it is essential to develop models accounting for the evolution of these microstructures. In this paper, we will perform a mathematical analysis of a new model, the so-called Phase Field Crystal modeling, introduced recently by K. Elder and M. Grant in [EG04].

This model describes for example liquid/solid phase transition, crystal growth or freezing (see $\left.\left[\mathrm{EPB}^{+} 07\right]\right)$. It represents an alternative to the usual approaches, namely

- the atomistic models: molecular dynamics, Boltzmann's equation, Monte-Carlo method;

- the continuum or phase field models: Cahn-Hilliard and Allen-Cahn equations, ...

These powerful methods have permitted to simulate many physical phenomenon: molecular motions, spacial structure in molecular liquids, spinodal decomposition, orderdisorder transition kinetics, ... However, the former applies on very short time scales and the latter gives rise to spatially uniform fields at equilibrium.

The Phase Field Crystal method extends the phase field modeling and allows to simulate periodic microstructure on large time scale. More precisely, as explained in [EG04], "the field to be considered (the time-average density) is only averaged in time and not in space", contrary to standard phase field models. Furthermore, the model is constructed to produce fields that are periodic in space by considering free energies involving second order derivatives. In a liquid/solid system, the solid phase is represented by a periodic field whose wavelength accounts for the distance between neighbouring atoms. The liquid state is described by a (spatially) uniform field. We refer the reader to $\left[\mathrm{EG} 04, \mathrm{EPB}^{+} 07, \mathrm{PDA}^{+} 07\right.$ ] for a more comprehensive exposition of the Phase Field Crystal method.

The simplest Phase Field Crystal model is the following sixth order evolution equation:

$$
\partial_{t} u-\partial_{x x}\left(\partial_{x x x x} u+2 \partial_{x x} u+f(u)\right)=0, \quad t>0, x \in(0, L),
$$

Date: July 22, 2009.

2000 Mathematics Subject Classification. 35Q20, 35J40, 35B32.

Key words and phrases. Phase Field Crystal, Bifurcation Theory, Higher Order Elliptic Equations. 
where $L$ is the length of the domain and where $f$ is the derivative of a double-well potential. This equation can be viewed as a conservative Swift-Hohenberg equation (see for instance [PR03, PR04, PW07, VdBPT01]) exactly as the Cahn-Hilliard equation is a conservative version of the Allen-Cahn equation. Performing a linear change of variable mapping $(0, L)$ onto $(0,1)$, equation $(1.1)$ can be rewritten

$$
\partial_{t} u-\varepsilon \partial_{x x}\left(\varepsilon^{2} \partial_{x x x x} u+2 \varepsilon \partial_{x x} u+f(u)\right)=0, \quad t>0, x \in(0,1),
$$

with $\varepsilon=1 / L^{2}$.

In this paper, we will focus on the stationary solutions to (1.2) complemented with initial and boundary conditions (see (2.7)). From a physical point of view, the main questions we would like to address are

- How can this equation produce stable periodic solutions? What is their wavelength ? - Does there exist stable equilibria to (1.2) that represent phase coexistence in a liquid/solid system?

By using bifurcation technics for theoretical results and for numerical simulations as well, we bring the following answers to these questions.

- There exist bifurcation points $(u, \varepsilon)=\left(M, \varepsilon_{*}\right)$ such that if $(u, \varepsilon)$ is a non trivial stationary solution to $(2.7)$ close to $\left(M, \varepsilon_{*}\right)$ then $u$ is a $2 \pi c_{f} \sqrt{\varepsilon_{*}}$-periodic function where $c_{f}=\left(1 \pm \sqrt{1-f^{\prime}(M)}\right)^{-1 / 2}$. Moreover, if the trivial solution $(M, \varepsilon)$ is stable for $\varepsilon<\varepsilon_{*}$ or $\varepsilon>\varepsilon_{*}$ then the latter periodic function $u$ is stable according to Theorems 5.5 and 5.8. If $(M, \varepsilon)$ is unstable for $\varepsilon$ close $\varepsilon_{*}, u$ is also unstable. By symmetry, we prove that $u\left(\cdot+\pi c_{f} \sqrt{\varepsilon_{*}}\right)$ is also a solution.

- As predicted by the thermodynamics, we are able to exhibit stable stationary solutions to the Phase Field Crystal equation that are a mixture of constant and periodic solutions: see Figures 16 and 17. These solutions can indeed be interpreted as representing phase coexistence in a liquid/solid system. They are obtained numerically by using a path-following method.

From a mathematical point of view, we would like to describe the solutions to (1.2). For this, we use local bifurcation theory to get multiplicity results and asymptotic expansion of bifurcating solutions (see Theorem 5.2, 5.7 and 5.11 ). In Theorem 5.3, we determine the shape of the bifurcation branches near bifurcation points. We prove, in Theorem 5.5, that the Principle of Exchange of Stability holds in the sense of Definition 5.2. We compare, for fixed $\varepsilon$, the energy of the bifurcating solutions with the energy of the trivial solution (see Theorem 5.6).

Our analysis relies on the Lyapunov-Schmidt method (see for instance [Kie04, CH82, AP93]). In some critical cases (Subsection 5.1 and 5.3), we use in addition Newton polygon's method.

Since our analytical results are essentially local (i.e. valid close to bifurcation points), we complete the description of the solution set to (1.2) by numerical simulations. We use also a bifurcation approach to track non trivial solutions (see Section 6). The Morse index of each solution is computed and is represented on bifurcation diagrams by specific color, as for example in Figure 5. These numerical simulations highlight a broad variety of bifurcation branches: bounded branches connecting two bifurcation points, loops, branches with complicated geometry (Figure 7), bifurcations from non trivial solution (Figure 15).

In the next three sections, we introduce some notations and we give preliminary results. Section 5 is devoted to theoretical bifurcation analysis. Finally, Section 6 deals with numerical simulations. 


\section{Equations and Functional Setting}

Let $\Omega=(0,1) \subset \mathbb{R}, \varepsilon>0$ and $r$ be a real parameter. We define

$$
\begin{aligned}
f & : \mathbb{R} \rightarrow \mathbb{R}, \quad u \mapsto(1+r) u+u^{3} \\
W_{0} & =\left\{u \in H^{2}(\Omega) \mid u^{\prime}(0)=u^{\prime}(1)=0\right\} \\
V_{0} & =\left\{u \in H^{4}(\Omega) \mid u^{\prime}=u^{\prime \prime \prime}=0 \text { on } \partial \Omega\right\} \\
\dot{L}^{2}(\Omega) & =\left\{v \in L^{2}(\Omega) \mid \int_{\Omega} v \mathrm{~d} x=0\right\} \\
\dot{V}_{0} & =V_{0} \cap \dot{L}^{2}(\Omega), \quad \dot{W}_{0}=W_{0} \cap \dot{L}^{2}(\Omega) .
\end{aligned}
$$

The space $\dot{V}_{0}$ is equipped with the bilinear form

$$
(u, v)_{\dot{V}_{0}}=\int_{\Omega} u^{(4)} v^{(4)} \mathrm{d} x
$$

which becomes in turn a Hilbert space since every $v \in \dot{V}_{0}$ satisfies

$$
\|v\|_{2} \leq \frac{1}{\lambda_{1}^{2}}\left\|v^{(4)}\right\|_{2}
$$

where $\|\cdot\|_{2}$ denotes the $L^{2}(\Omega)$-norm. Indeed,

$$
\|v\|_{2} \leq \frac{1}{\sqrt{\lambda_{1}}}\left\|v^{\prime}\right\|_{2} \leq \frac{1}{\lambda_{1}}\left\|v^{\prime \prime}\right\|_{2}
$$

by Poincaré-Wirtinger and Poincaré's inequalities. Here $\lambda_{1}:=\pi^{2}$ denotes the first eigenvalue of the one-dimensional Laplace operator with homogeneous Dirichlet boundary conditions on $\Omega$. Moreover $v^{\prime \prime}$ belongs to $\dot{W}_{0}$ thus the same estimates give $\left\|v^{\prime \prime}\right\|_{2} \leq \frac{1}{\lambda_{1}}\left\|v^{(4)}\right\|_{2}$. Then (2.5) follows. In the same way, if $(u, v)_{\dot{W}_{0}}:=\int_{\Omega} u^{(2)} v^{(2)} \mathrm{d} x$ then $\left(\dot{W}_{0},(\cdot, \cdot)_{\dot{W}_{0}}\right)$ is a Hilbert space. Of course, $u^{(2)}$ stands for $\partial_{x x} u$.

Given initial data $u_{0}=u_{0}(x)$, the Phase Field Crystal equation with homogeneous Neuman boundary condition reads

$$
\left\{\begin{aligned}
\partial_{t} u-\varepsilon \partial_{x x}\left(\varepsilon^{2} \partial_{x x x x} u+2 \varepsilon \partial_{x x} u+f(u)\right) & =0 & & \text { in } \Omega \times(0, \infty) \\
u^{\prime}=u^{(3)}=u^{(5)} & =0 & & \text { on } \partial \Omega \times(0, \infty) \\
u(0) & =u_{0} & & \text { in } \Omega .
\end{aligned}\right.
$$

Since every solution to (2.7) satisfies

$$
\int_{\Omega} u(x, t) \mathrm{d} x=\int_{\Omega} u_{0}(x) \mathrm{d} x \quad \forall t>0
$$

the stationary solutions to the problem above solve

$$
u \in M+\dot{V}_{0}, \quad \varepsilon^{2} u^{(4)}+2 \varepsilon u^{(2)}+f(u)=\int_{\Omega} f(u) \mathrm{d} x \quad \text { in } L^{2}(\Omega),
$$

where $M:=\int_{\Omega} u_{0}(x)$ is a real parameter. Associated to this equation is the energy functional

$$
E: W_{0} \times(\varepsilon, \infty) \rightarrow \mathbb{R}, \quad(u, \varepsilon) \mapsto \int_{\Omega} \frac{1}{2}\left(\varepsilon u^{\prime \prime}+u\right)^{2}+\frac{r}{2} u^{2}+\frac{1}{4} u^{4} \mathrm{~d} x .
$$


Introducing the new function $v$ defined by $u=M+v,(2.8)$ is equivalent to

$$
v \in \dot{V}_{0}, \quad \varepsilon^{2} v^{(4)}+2 \varepsilon v^{(2)}+f(M+v)=\int_{\Omega} f(M+v) \mathrm{d} x \quad \text { in } \quad L^{2}(\Omega),
$$

or by Taylor's expansion of $f(M+v)$, to

$$
\left\{\begin{aligned}
v \in \dot{V}_{0}, \quad \varepsilon^{2} v^{(4)}+2 \varepsilon v^{(2)} & +f^{\prime}(M) v+\frac{f^{\prime \prime}(M)}{2}\left(v^{2}-\int_{\Omega} v^{2} d x\right) \\
& +\frac{f^{\prime \prime \prime}(M)}{6}\left(v^{3}-\int_{\Omega} v^{3} d x\right)=0 \quad \text { in } L^{2}(\Omega) .
\end{aligned}\right.
$$

We split the right hand side of (2.11) into linear and nonlinear parts by setting

$$
\begin{aligned}
L(\cdot, \varepsilon): \dot{V}_{0} & \rightarrow \dot{L}^{2}(\Omega), \quad v \mapsto \varepsilon^{2} v^{(4)}+2 \varepsilon v^{(2)}+f^{\prime}(M) v \\
N: \dot{V}_{0} & \rightarrow \dot{L}^{2}(\Omega), \quad v \mapsto \frac{f^{\prime \prime}(M)}{2}\left(v^{2}-\int_{\Omega} v^{2} d x\right)+\frac{f^{\prime \prime \prime}(M)}{6}\left(v^{3}-\int_{\Omega} v^{3} d x\right) .
\end{aligned}
$$

Finally we define

$$
F: \dot{V}_{0} \times(0, \infty) \rightarrow \dot{L}^{2}(\Omega), \quad v \mapsto L(v, \varepsilon)+N(v),
$$

so what instead of (2.10), we will consider the bifurcation problem

$$
v \in \dot{V}_{0}, \varepsilon>0, \quad F(v, \varepsilon)=0 \quad \text { in } \dot{L}^{2}(\Omega)
$$

\section{Preliminary Results}

Proposition 3.1. For every positive $\varepsilon$, the energy $E(\cdot, \varepsilon)$ admits a minimizer on $M+\dot{V}_{0}$. Moreover, if

$$
E(u, \varepsilon)<E(M, \varepsilon)
$$

for some $u \in M+\dot{W}_{0}$, then (2.8) admits at least two solutions.

Proof. For every positive $\varepsilon, E(\cdot, \varepsilon)$ is coercive and weakly sequentially lower semicontinuous on $M+\dot{W}_{0}$. Then it is well know that $E(\cdot, \varepsilon)$ admits a minimizer $u_{0}$ on $M+\dot{W}_{0}$. By Fourier multiplier theory, $u_{0}$ is solution to

$$
\left\{\begin{array}{l}
\text { Find } u \in M+\dot{W}_{0} \text { such that for every } \varphi \in W_{0} \\
\int_{\Omega} \varepsilon^{2} u^{\prime \prime} \varphi^{\prime \prime}-2 \varepsilon u^{\prime} \varphi^{\prime}+f(u) \varphi \mathrm{d} x=\int_{\Omega} f(u) \mathrm{d} x \int_{\Omega} \varphi \mathrm{d} x .
\end{array}\right.
$$

A bootstrap argument shows that $u_{0}$ is in $M+\dot{V}_{0}$. Hence $u_{0}$ is a minimizer on $M+\dot{V}_{0}$ and solves (2.8). The second assertion is then obvious.

We now give an uniform $L^{\infty}$ bound for solutions to (2.10).

Proposition 3.2. There exists a constant $C$ depending only on $r$ and $M$ such that for every solution $v$ to $(2.10)$,

$$
\|v\|_{\infty} \leq \frac{C}{\varepsilon} .
$$

Proof. Since $\int_{\Omega} v=0$, there exists $x_{0} \in \Omega$ such that $v\left(x_{0}\right)=0$. Then, for each $x \in \Omega$, the Cauchy-Schwarz inequality and (2.6) lead to

$$
|v(x)|=\left|\int_{x_{0}}^{x} v^{\prime}(s) \mathrm{d} s\right| \leq\left|x-x_{0}\right|^{1 / 2}\left\|v^{\prime}\right\|_{2} \leq\left\|v^{\prime}\right\|_{2} \leq \frac{1}{\sqrt{\lambda_{1}}}\left\|v^{\prime \prime}\right\|_{2} .
$$


Hence it is enough to estimate $\left\|v^{\prime \prime}\right\|_{2}$. For this, we first notice that for some positive constants $c_{1}, C_{1}$, there holds

$$
\begin{aligned}
& f(u) u \geq c_{1} u^{4}-C_{1} \\
& |f(u)| \leq C_{1}\left(|u|^{3}+1\right) \quad \forall u \in \mathbb{R} .
\end{aligned}
$$

Then, for all $v \in \mathbb{R}$,

$$
f(M+v) v \geq \frac{c_{1}}{2} v^{4}-C,
$$

where $C$ is positive and depends only on $r$ and $M$. Now testing (2.10) with $\varphi=v$ and using (3.2), we obtain

$$
\varepsilon^{2}\left\|v^{\prime \prime}\right\|_{2}^{2}+\frac{c_{1}}{2} \int_{\Omega} v^{4} \mathrm{~d} x \leq 2 \varepsilon\left\|v^{\prime}\right\|_{2}^{2}+C .
$$

Furthermore, $\left\|v^{\prime}\right\|_{2}^{2}=-\int_{\Omega} v v^{\prime \prime}$ and for every $a, b, \delta>0$, we have the Young inequality

Thus we deduce

$$
a b \leq \frac{\delta}{4} a^{4}+\frac{3}{4 \delta^{1 / 3}} b^{4 / 3} .
$$

$$
2 \varepsilon\left\|v^{\prime}\right\|_{2}^{2} \leq \frac{\varepsilon \delta}{2} \int_{\Omega} v^{4} \mathrm{~d} x+\frac{3 \varepsilon}{2 \delta^{1 / 3}} \int_{\Omega}\left|v^{\prime \prime}\right|^{4 / 3} \mathrm{~d} x .
$$

By choosing $\delta=c_{1} / \varepsilon$ and going back to (3.3), we deduce with Hölder's inequality,

$$
\left(\varepsilon\left\|v^{\prime \prime}\right\|_{2}\right)^{2} \leq \frac{3}{2 c_{1}^{1 / 3}}\left(\varepsilon\left\|v^{\prime \prime}\right\|_{2}\right)^{4 / 3}+C .
$$

Hence (3.1) follows.

For large $\varepsilon,(2.8)$ and (2.10) possess an unique solution. More precisely, the following result holds.

Proposition 3.3. If $r \geq 0$ then (2.10) has a unique solution. If $r<0$ then (2.10) has a unique solution provided $\varepsilon>\varepsilon_{0}$ where

$$
\varepsilon_{0}=\frac{1+\sqrt{-r}}{\lambda_{1}}
$$

We recall that $\lambda_{1}:=\pi^{2}$ denotes the first eigenvalue of the one-dimensional Laplace operator with homogeneous Dirichlet boundary conditions on $\Omega$.

Proof. Let $u$ and $v$ be two solutions of (2.10) and $g$ be the function defined by $g(u)=$ $f(u)-u$. Then testing the difference of the two equations with $u-v$, we find

$$
\int_{\Omega}\left(\varepsilon(u-v)^{\prime \prime}+u-v\right)^{2} \mathrm{~d} x+\int_{\Omega}(g(u)-g(v))(u-v) \mathrm{d} x=0 .
$$

Moreover,

$$
(g(u)-g(v))(u-v) \geq r(u-v)^{2}+\left(u^{3}-v^{3}\right)(u-v) .
$$

Thus,

$$
\left\|\varepsilon(u-v)^{\prime \prime}+u-v\right\|_{2}^{2}+r\|u-v\|_{2}^{2}+\int_{\Omega}\left(u^{3}-v^{3}\right)(u-v) d x \leq 0 .
$$

Then $u=v$ in the case $r \geq 0$. Otherwise, by the triangular inequality,

$$
\varepsilon\left\|(u-v)^{\prime \prime}\right\|_{2} \leq(1+\sqrt{-r})\|u-v\|_{2} .
$$

By using (2.6), we conclude that $u=v$ if $\varepsilon>\varepsilon_{0}$, where $\varepsilon_{0}$ is given by (3.4). 
Remark 3.1. According to [EG04], the parameter $r$ is proportional to the temperature of the system.

\section{The Linearized Equation}

We study the eigenvalue problem (see (2.3), (2.12) for notation)

$$
\left\{\begin{array}{l}
L(\varphi, \varepsilon)=\lambda \varphi \quad \text { in } L^{2}(\Omega) \\
\varphi \in \dot{V}_{0} \backslash\{0\}, \lambda \in \mathbb{R} .
\end{array}\right.
$$

Under the notation of Section 2, we put $p:=1-f^{\prime}(M)$ and $\lambda_{k}:=(k \pi)^{2}$ for $k=1,2, \ldots$ It is easy to see that the eigenvalues of (4.1) are of the form

$$
\left(\varepsilon \lambda_{k}-1\right)^{2}-p, \quad k=1,2, \ldots,
$$

with corresponding eigenfunctions

$$
\varphi_{k}: \Omega \rightarrow \mathbb{R}, \quad x \mapsto \cos (k \pi x) .
$$

For $k \geq 1$ and $p \geq 0$, we put

$$
\varepsilon_{k}:=\frac{1+\sqrt{p}}{\lambda_{k}}, \quad \bar{\varepsilon}_{k}:=\frac{1-\sqrt{p}}{\lambda_{k}} .
$$

Then the following description of $\operatorname{ker} L(\cdot, \varepsilon)$ holds.

Proposition 4.1. Let $M \in \mathbb{R}, p:=1-f^{\prime}(M)$ and $\varepsilon>0$.

- If $p \in\{0\} \cup[1, \infty)$ and there exists $k \geq 1$ such that $\varepsilon=\varepsilon_{k}$ then

$$
\operatorname{ker} L(\cdot, \varepsilon)=\left\langle\varphi_{k}\right\rangle \text {. }
$$

- If $0<p<1$ and there exists $k \geq 1$ such that $\varepsilon=\varepsilon_{k}$ then

- if $p \in\left\{p_{k, k^{\prime}} \mid k^{\prime}=1, \ldots, k-1\right\}$ (see (4.5) below for the definition of $\left.p_{k, k^{\prime}}\right)$ then

$$
\operatorname{ker} L(\cdot, \varepsilon)=\left\langle\varphi_{k}, \varphi_{k^{\prime}}\right\rangle \text {; }
$$

- if $p \notin\left\{p_{k, k^{\prime}} \mid k^{\prime}=1, \ldots, k-1\right\}$ then $\operatorname{ker} L(\cdot, \varepsilon)=\left\langle\varphi_{k}\right\rangle$.

- In any other case, $\operatorname{ker} L(\cdot, \varepsilon)=\{0\}$.

Proof. We will prove only the case where $p \in(0,1)$. For $k \geq 1$, we put

$$
\varepsilon_{k}:=\frac{1+\sqrt{p}}{\lambda_{k}}
$$

and for $k^{\prime}=1, \ldots, k-1$, we denote by $p_{k, k^{\prime}}$ the unique solution in $(0,1)$ of

$$
\frac{\lambda_{k}}{\lambda_{k^{\prime}}}=\frac{1+\sqrt{p_{k, k^{\prime}}}}{1-\sqrt{p_{k, k^{\prime}}}} \text {. }
$$

If $p=p_{k, k^{\prime}}$ then $\varepsilon_{k} \lambda_{k^{\prime}}=1-\sqrt{p_{k, k^{\prime}}}$, hence $L\left(\varphi_{k^{\prime}}, \varepsilon_{k}\right)=0$ and $\operatorname{ker} L(\cdot, \varepsilon)$ is generated by $\varphi_{k}$ and $\varphi_{k^{\prime}}$. The remainder of the proof is left to the reader.

The linear stability of the trivial solution to (2.10) is given by the following statement.

Proposition 4.2. Let $p:=1-f^{\prime}(M)$ and suppose that 0 is not in the spectrum of $L(\cdot, \varepsilon)$. Besides,

- if $0<p<1$ then let

$$
k_{+}:=\left[-\frac{1}{2}+\frac{1+\sqrt{1-p}}{2 \sqrt{p}}\right]+1
$$


be the smallest integer larger than the number into brackets. If

$$
\varepsilon \in\left(0, \varepsilon_{k_{+}}\right) \dot{\cup}\left(\bar{\varepsilon}_{k_{+}-1}, \varepsilon_{k_{+}-1}\right) \dot{\cup} \ldots \dot{\cup}\left(\bar{\varepsilon}_{2}, \varepsilon_{2}\right) \dot{\cup}\left(\bar{\varepsilon}_{1}, \varepsilon_{1}\right)
$$

then the trivial solution to (2.10) is unstable.

- If $p \geq 1$ and $\varepsilon<\varepsilon_{1}$ then $v=0$ is also unstable.

- Otherwise $v=0$ is stable.

Proof. By (4.2), we have to assess the sign of $\left(\varepsilon \lambda_{k}-1\right)^{2}-p$ for each $k \geq 1$, assuming that it is non zero. More precisely, $v=0$ is unstable if and only if there exists some integer $k$ such that $\left(\varepsilon \lambda_{k}-1\right)^{2}-p<0$.

Hence, we first observe that 0 is stable if $p<0$. If $p \geq 1$ then $\bar{\varepsilon}_{1} \leq 0$ hence if $\varepsilon<\varepsilon_{1}$ then $v=0$ is unstable. If $p \geq 0$ and $\varepsilon>\varepsilon_{1}$ then for every $k \geq 1$,

$$
\varepsilon \lambda_{k}-1>\varepsilon_{1} \lambda_{1}-1=\sqrt{p} .
$$

Hence $\left(\varepsilon \lambda_{k}-1\right)^{2}-p>0$ and $v=0$ is stable. There remains to consider the case where $0<p<1$ and $\varepsilon<\varepsilon_{1}$. Recalling (4.4), we have clearly

$$
\bar{\varepsilon}_{k} \leq \varepsilon_{k} \quad \text { and } \quad \varepsilon_{k+1} \leq \varepsilon_{k} .
$$

Thus $\varepsilon \in\left(\bar{\varepsilon}_{k}, \varepsilon_{k}\right)$ yields the instability of 0 . Moreover, $\bar{\varepsilon}_{k} \leq \varepsilon_{k+1}$ if and only if $k \geq k_{+}$. This completes the proof of the theorem.

Remark 4.1. - If $M=0$ and $r<0$ then $\varepsilon_{1}$ and the solution $\varepsilon_{0}$ to (3.4) coincide. According to Propositions 3.1, 3.3 and 4.2, if we suppose in addition that $-1<r<0$ then $(2.10)$ admits an unique solution for $\varepsilon \in\left(\varepsilon_{1}, \infty\right)$; at least two solutions for $\varepsilon \in\left(\bar{\varepsilon}_{1}, \varepsilon_{1}\right)$.

- $k_{+}=1 \Longleftrightarrow p>9 / 25$. Hence if $p>9 / 25$ then $\varepsilon_{k_{+}}=\varepsilon_{1}$ and $v=0$ is unstable for $\varepsilon \in\left(0, \varepsilon_{1}\right)$.

- The case $0<p<1$ and $k_{+}=3$ is depicted in Fig. 1 .

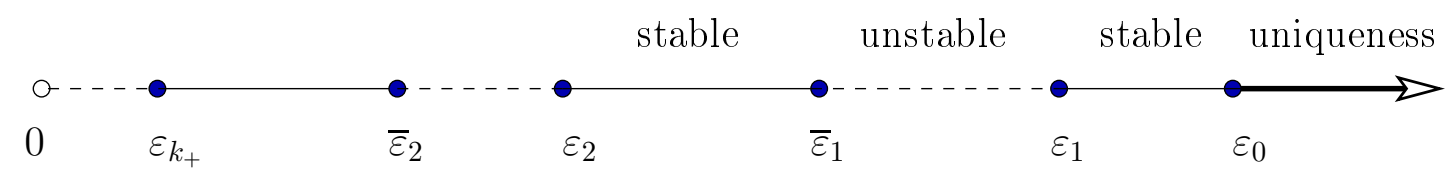

FiguRE 1. Stability intervals w.r.t. $\varepsilon$ when $k_{+}=3$

- Proposition 4.2 states the stability analysis in terms of $\varepsilon$ when $p$ is given. However, we may fix $\varepsilon$ first and discuss the stability of the trivial solution with respect to $p$. For example, let us choose $\varepsilon$ so what $2 / \lambda_{2}<\varepsilon<1 / \lambda_{1}$. In order to emphasize the dependence on $p$, we will denote $\varepsilon_{k}$ by $\varepsilon_{k}(p)$. If $p \in(0,1)$ then

$$
\varepsilon_{2}(p)<\varepsilon<1 / \lambda_{1}=\bar{\varepsilon}_{1}(0) .
$$

According to Proposition 4.2, $v=0$ is unstable if and only if $\varepsilon>\bar{\varepsilon}_{1}(p)$ i.e. $p>\left(1-\varepsilon \lambda_{1}\right)^{2}$. Since $p=-\left(r+3 M^{2}\right)$, the phase diagram in the plan $(M, r)$ is determined by the parabola

$$
r=-3 M^{2}-\left(1-\varepsilon \lambda_{1}\right)^{2} .
$$

Indeed, if $r<(>)-3 M^{2}-\left(1-\varepsilon \lambda_{1}\right)^{2}$ then $v=0$ is unstable (stable). 


\section{Bifurcation Analysis}

According to the Implicit Function Theorem, bifurcation from the trivial solution occurs at $\left(M, \varepsilon_{*}\right)$ only if the kernel of $L\left(\cdot, \varepsilon_{*}\right)$ is non trivial. We will first assume that it is one-dimensional. More precisely with the above notation (in particular (2.12), (4.3)), we suppose

$$
p:=1-f^{\prime}(M)>0
$$

and that there exists a positive integer $k_{*}$ such that if

$$
\varepsilon_{*}:=\frac{1 \pm \sqrt{p}}{\lambda_{k_{*}}}>0
$$

then

$$
\operatorname{ker} L\left(\cdot, \varepsilon_{*}\right)=\left\langle\varphi_{k_{*}}\right\rangle .
$$

Recall that Proposition 4.1 gives necessary and sufficient conditions for (5.3) to hold in terms of the data of the problem. For simplicity we will put

$$
\varphi_{*}=\varphi_{k_{*}}, \quad \lambda_{*}=\lambda_{k_{*}} .
$$

Our main tool for the analysis of bifurcations is the Lyapunov-Schmidt method (see for instance [Kie04, CH82, AP93]). Since the operator $L\left(\cdot, \varepsilon_{*}\right)$ is self-adjoint with compact resolvent, the set

$$
\left\{\frac{\varphi_{k}}{\left\|\varphi_{k}\right\|_{2}} \mid k \in \mathbb{N} \backslash\{0\}\right\}
$$

is a spectral basis in $\dot{L}^{2}(\Omega)$. Thus

$$
\operatorname{dim} \operatorname{ker} L\left(\cdot, \varepsilon_{*}\right)=\operatorname{codim} R\left(L\left(\cdot, \varepsilon_{*}\right)\right) \text {, }
$$

where $R\left(L\left(\cdot, \varepsilon_{*}\right)\right) \subset \dot{L}^{2}(\Omega)$ denotes the range of $L\left(\cdot, \varepsilon_{*}\right)$, and

$$
\begin{aligned}
\dot{L}^{2}(\Omega) & =R\left(L\left(\cdot, \varepsilon_{*}\right)\right) \oplus \operatorname{ker} L\left(\cdot, \varepsilon_{*}\right) \\
\dot{V}_{0} & =\left(R\left(L\left(\cdot, \varepsilon_{*}\right)\right) \cap \dot{V}_{0}\right) \oplus \operatorname{ker} L\left(\cdot, \varepsilon_{*}\right) .
\end{aligned}
$$

The decomposition of $\dot{L}^{2}(\Omega)$, in turn, defines the projections

$$
\begin{aligned}
& Q: \dot{L}^{2}(\Omega) \rightarrow R\left(L\left(\cdot, \varepsilon_{*}\right)\right) \quad \text { along } \operatorname{ker} L\left(\cdot, \varepsilon_{*}\right) \\
& P:=I-Q: \dot{L}^{2}(\Omega) \rightarrow \operatorname{ker} L\left(\cdot, \varepsilon_{*}\right) .
\end{aligned}
$$

Denoting by $(\cdot, \cdot)_{2}$ the $L^{2}$-scalar product, there holds

$$
P v=\frac{\left(v, \varphi_{*}\right)_{2}}{\left\|\varphi_{*}\right\|_{2}^{2}} \varphi_{*}=2\left(v, \varphi_{*}\right)_{2} \varphi_{*}, \quad \forall v \in \dot{L}^{2}(\Omega) .
$$

In the sequel, we will sometimes identify the vector $P v$ with its coordinate in the basis $\varphi_{*}$; namely we will write $P v=2\left(v, \varphi_{*}\right)_{2}$.

We decompose every $v \in \dot{V}_{0}$ in a unique way into

$$
v=y \varphi_{*}+w_{2},
$$

where $y \in \mathbb{R}$ and $w_{2}$ belongs to $\varphi_{*}^{\perp}$, the orthogonal space of $\varphi_{*}$ in $\dot{V}_{0}$. Integrations by parts yield

$$
\varphi_{*}^{\perp}=\left\{v \in \dot{V}_{0} \mid\left(v, \varphi_{*}\right)_{2}=0\right\} .
$$

Then the solutions to (2.14) are given by

$$
y \in \mathbb{R}, w_{2} \in \varphi_{*}^{\perp}, \varepsilon>0, \quad y L\left(\varphi_{*}, \varepsilon\right)+L\left(w_{2}, \varepsilon\right)+N\left(y \varphi_{*}+w_{2}\right)=0 .
$$


Projection on $\boldsymbol{R}\left(\boldsymbol{L}\left(\cdot, \boldsymbol{\varepsilon}_{*}\right)\right)$. Applying $Q$ to $(5.9)$, we obtain

$$
y Q L\left(\varphi_{*}, \varepsilon\right)+Q L\left(w_{2}, \varepsilon\right)+Q N\left(y \varphi_{*}+w_{2}\right)=0 \quad \text { in } \dot{L}^{2}(\Omega) .
$$

By the Implicit Function Theorem, for $\left(y, \varepsilon, w_{2}\right)$ close to $\left(0, \varepsilon_{*}, 0\right)$, this equation is equivalent to

$$
w_{2}=w_{2}(y, \varepsilon), \quad w_{2}\left(0, \varepsilon_{*}\right)=0,
$$

where $w_{2}(\cdot, \cdot)$ is defined and smooth from some neighbourhood of $\left(0, \varepsilon_{*}\right)$ into some neighbourhood of 0 in $R\left(L\left(\cdot, \varepsilon_{*}\right)\right.$ ) (see for instance [Kie04, Section I-2]). Next, letting $w_{2}=w_{2}(y, \varepsilon)$ in $(5.10)$ and differentiating w.r.t $y$, we obtain $\partial_{y} w_{2}\left(0, \varepsilon_{*}\right)=0$. Moreover, since $w_{2}(y, \varepsilon)$ is the unique local solution to (5.10), it results that $w_{2}(0, \varepsilon)=0$ for $\varepsilon$ close to $\varepsilon_{*}$. Hence, we may write $w_{2}$ under the form

$$
w_{2}(y, \varepsilon)=y v_{2}(y, \varepsilon),
$$

where the new function $v_{2}$, defined by

$$
v_{2}(y, \varepsilon):=\int_{0}^{1} \partial_{y} w_{2}(y t, \varepsilon) \mathrm{d} t
$$

satisfies $v_{2}\left(0, \varepsilon_{*}\right)=0$ since $\partial_{y} w_{2}\left(0, \varepsilon_{*}\right)=0$. By setting,

$$
\bar{N}(v, y):=\frac{f^{\prime \prime}(M)}{2}\left(v^{2}-\int_{\Omega} v^{2} d x\right)+y \frac{f^{\prime \prime \prime}(M)}{6}\left(v^{3}-\int_{\Omega} v^{3} d x\right),
$$

we have $N(y v)=y^{2} \bar{N}(v, y)$. Then $(5.10),(5.12)$ yield

$$
Q L\left(\varphi_{*}, \varepsilon\right)+Q L\left(v_{2}(y, \varepsilon), \varepsilon\right)+y \bar{N}\left(\varphi_{*}+v_{2}(y, \varepsilon), y\right)=0 .
$$

By differentiating this equation w.r.t. $y$, evaluating at $(y, \varepsilon)=\left(0, \varepsilon_{*}\right)$ and using the fact that $\bar{N}\left(\varphi_{*}, 0\right)=f^{\prime \prime}(M) / 2\left(\varphi_{*}^{2}-\int_{\Omega} \varphi_{*}^{2}\right)$ belongs to $\varphi_{*}^{\perp}$, we obtain that $w:=\partial_{y} v_{2}\left(0, \varepsilon_{*}\right)$ is solution to

$$
L\left(w, \varepsilon_{*}\right)+\frac{f^{\prime \prime}(M)}{2}\left(\varphi_{*}^{2}-\int_{\Omega} \varphi_{*}^{2}\right)=0 \quad \text { in } \dot{L}^{2}(\Omega) .
$$

For later use, we will compute $w$. Testing (5.15) with $\varphi_{k}$ for $k \neq k_{*}$, we get

$$
\int_{\Omega} w L\left(\varphi_{k}, \varepsilon_{*}\right) \mathrm{d} x+\frac{f^{\prime \prime}(M)}{2} \int_{\Omega} \varphi_{*}^{2} \varphi_{k} \mathrm{~d} x=0 .
$$

Since $\varphi_{k}$ is an eigenfunction of $L\left(\cdot, \varepsilon_{*}\right)$ with eigenvalue $\left(\varepsilon_{*} \lambda_{k}-1\right)^{2}-p$, which is non zero by (5.3), we obtain using also $\varphi_{*}^{2}=\frac{1}{2} \varphi_{2 k_{*}}+\frac{1}{2}$,

$$
\int_{\Omega} w \varphi_{k} \mathrm{~d} x=\frac{-f^{\prime \prime}(M) / 4}{\left(\varepsilon_{*} \lambda_{k}-1\right)^{2}-p} \int_{\Omega} \varphi_{2 k_{*}} \varphi_{k} \mathrm{~d} x .
$$

Moreover, $\left(\varphi_{2 k_{*}}, \varphi_{k}\right)_{2}=\frac{1}{2} \delta_{2 k_{*}, k}$, thus

$$
w=\partial_{y} v_{2}\left(0, \varepsilon_{*}\right)=\frac{-f^{\prime \prime}(M) / 4}{\left(\varepsilon_{*} \lambda_{2 k_{*}}-1\right)^{2}-p} \varphi_{2 k_{*}} .
$$

Projection on $\left\langle\boldsymbol{\varphi}_{\boldsymbol{k}_{*}}\right\rangle$. By setting, in view of (5.5),

$$
g(y, \varepsilon):=P L\left(\varphi_{*}, \varepsilon\right)+P L\left(v_{2}(y, \varepsilon), \varepsilon\right)+y P \bar{N}\left(\varphi_{*}+v_{2}(y, \varepsilon), y\right),
$$

(5.9) and (5.12) yield

$$
g(y, \varepsilon)=0 \quad \text { in } \dot{L}^{2}(\Omega) .
$$


Since $\varepsilon_{*}^{2} \varphi_{*}^{(4)}+2 \varepsilon_{*} \varphi_{*}^{(2)}+f^{\prime}(M) \varphi_{*}=0$, we get with (5.6),

$$
\partial_{\varepsilon} g\left(0, \varepsilon_{*}\right)=2 \frac{\varepsilon_{*} \lambda_{*}-f^{\prime}(M)}{\varepsilon_{*}} \varphi_{*} .
$$

Moreover $\partial_{y} g\left(0, \varepsilon_{*}\right)=0$ (use $P \bar{N}\left(\varphi_{*}, 0\right)=0$ ) and the second derivative is given in the following Lemma.

Lemma 5.1. With the notations above, in particular (5.12) and (5.17), assume that $p \geq 0$ and (5.2), (5.3) hold. Then

$$
\begin{aligned}
\partial_{y}^{2} g\left(0, \varepsilon_{*}\right) & =4 f^{\prime \prime}(M) \int_{\Omega} \varphi_{*}^{2} \partial_{y} v_{2}\left(0, \varepsilon_{*}\right) \mathrm{d} x+\frac{2}{3} f^{\prime \prime \prime}(M) \int_{\Omega} \varphi_{*}^{4} \mathrm{~d} x \\
& =\frac{-f^{\prime \prime}(M)^{2} / 4}{\left(\varepsilon_{*} \lambda_{2 k_{*}}-1\right)^{2}-p}+f^{\prime \prime \prime}(M) / 4 .
\end{aligned}
$$

Proof. We compute $\partial_{y}^{2} g\left(0, \varepsilon_{*}\right)$ from (5.17) and use the relation

$$
\partial_{y}\left\{P \bar{N}\left(\varphi_{*}+v_{2}\left(y, \varepsilon_{*}\right), y\right)\right\}_{\mid y=0}=2 f^{\prime \prime}(M) \int_{\Omega} \varphi_{*}^{2} \partial_{y} v_{2}\left(0, \varepsilon_{*}\right) \mathrm{d} x+\frac{1}{3} f^{\prime \prime \prime}(M) \int_{\Omega} \varphi_{*}^{4} \mathrm{~d} x
$$

to get the first equality. Next, we obtain thanks to (5.16),

$$
\int_{\Omega} \partial_{y} v_{2}\left(0, \varepsilon_{*}\right) \varphi_{*}^{2} \mathrm{~d} x=\frac{-f^{\prime \prime}(M) / 4}{\left(\varepsilon_{*} \lambda_{2 k_{*}}-1\right)^{2}-p} \int_{\Omega} \varphi_{2 k_{*}} \varphi_{*}^{2} \mathrm{~d} x
$$

and the second equality follows since $\varphi_{*}^{2}=\frac{1}{2} \varphi_{2 k_{*}}+\frac{1}{2}$ and $\int_{\Omega} \varphi_{*}^{4} \mathrm{~d} x=3 / 8$.

Observe that $\partial_{\varepsilon} g\left(0, \varepsilon_{*}\right) \neq 0$ by (5.1), (5.2) and (5.19). Thus the Implicit Function Theorem yields that, for $(y, \varepsilon)$ close to $\left(0, \varepsilon_{*}\right),(5.18)$ is equivalent to

$$
\varepsilon=\varepsilon(y)=\varepsilon_{*}+O(y) \text {. }
$$

In particular,

$$
g(y, \varepsilon(y))=0 .
$$

Differentiating this equation and using $\partial_{y} g\left(0, \varepsilon_{*}\right)=0$, we obtain

$$
\dot{\varepsilon}(0)=0
$$

and, thanks to Lemma 5.1 and (5.19),

$$
\ddot{\varepsilon}(0)=-\frac{\partial_{y}^{2} g\left(0, \varepsilon_{*}\right)}{\partial_{\varepsilon} g\left(0, \varepsilon_{*}\right)}=\frac{\varepsilon_{*} / 8}{\varepsilon_{*} \lambda_{*}-f^{\prime}(M)}\left(\frac{f^{\prime \prime}(M)^{2}}{\left(\varepsilon_{*} \lambda_{2 k_{*}}-1\right)^{2}-p}-f^{\prime \prime \prime}(M)\right) .
$$

The following statement is typical from bifurcation results obtained by the Implicit Function Theorem.

Theorem 5.2. Assume that (5.1)-(5.3) hold. Then

- $\left(0, \varepsilon_{*}\right)$ is a bifurcation point for Equation (2.14);

- there exists locally a unique branch of non trivial solutions through $\left(0, \varepsilon_{*}\right)$;

- there exists a neighbourhood $\mathcal{N} \times I$ of $\left(0, \varepsilon_{*}\right)$ in $\dot{W}_{0} \times \mathbb{R}$, such that every solution to (2.14) in $\mathcal{N} \times I$ belongs to this branch.

Moreover, if

$$
\left(\varepsilon_{*} \lambda_{2 k_{*}}-1\right)^{2}-p \neq f^{\prime \prime 2} / f^{\prime \prime \prime}(M)
$$


then non trivial solutions $(v, \varepsilon)$ to $(2.14)$ exist in $\mathcal{N} \times I$ if and only if $\ddot{\varepsilon}(0)\left(\varepsilon-\varepsilon_{*}\right)>0$ where $\ddot{\varepsilon}(0)$ is given by (5.23). In that case, these solutions admit the following expansion

$$
v \simeq \pm \sqrt{\frac{2}{\tilde{\varepsilon}(0)}\left(\varepsilon-\varepsilon_{*}\right)} \varphi_{*} \quad \text { in } \dot{V}_{0} .
$$

Proof of Theorem 5.2. It follows from the above analysis. In particular, (5.14), (5.21) give local existence and uniqueness in $\dot{V}_{0} \times \mathbb{R}$ of the branch $\{(v(y), \varepsilon(y)) \mid y \in(-\delta, \delta)\}$ of non trivial solutions satisfying $v(0)=0$ and

$$
\varepsilon(y)=\varepsilon_{*}+\frac{\ddot{\varepsilon}(0)}{2} y^{2}+O\left(y^{3}\right) .
$$

If we want a result in the weaker topology of $\dot{W}_{0}$, we need to consider weak solutions to (2.8), as in the proof of Proposition 3.1. To be more specific, let $\dot{W}_{0}^{\prime}$ be the dual space of $\dot{W}_{0}$ and

$$
A: \dot{L}^{2}(\Omega) \rightarrow \dot{W}_{0}^{\prime}, \quad u \mapsto-u^{\prime \prime} .
$$

Then $\dot{W}_{0}^{\prime}$ eqquiped with the bilinear form

$$
(f, g)_{\dot{W}_{0}^{\prime}}=\left(A^{-1} f, A^{-1} g\right)_{2},
$$

is a Hilbert space. The above bifurcation analysis can be made in the spaces $\dot{W}_{0}, \dot{W}_{0}^{\prime}$ instead of $\dot{V}_{0}$ and $\dot{L}^{2}(\Omega)$. Besides, since strong solutions are weak solutions and the bifurcation branches are locally unique, we obtain the third assertion of the theorem. Finally, assuming $\ddot{\varepsilon}(0) \neq 0$ i.e. (5.24), we deduce the approximation (5.25).

Remark 5.1. - (5.25) means that, if $(v, \varepsilon)$ is a solution to $(2.14)$ then there exists $\delta \in$ $\{-1,1\}$ such that

$$
\left|\varepsilon-\varepsilon_{*}\right|^{-1 / 2}\left\|v-\delta \sqrt{\frac{2}{\tilde{\varepsilon}(0)}\left(\varepsilon-\varepsilon_{*}\right)} \varphi_{*}\right\|_{\dot{V}_{0}} \underset{\varepsilon \rightarrow \varepsilon_{*}}{\longrightarrow} 0 .
$$

- If we go back to the original unknown $u=M+v$ then Theorem 5.2 yields that $(2.8)$ has exactly three solutions in $M+\mathcal{N}$ if $\varepsilon \in I$ satisfies $\ddot{\varepsilon}(0)\left(\varepsilon-\varepsilon_{*}\right)>0$. Otherwise if $\ddot{\varepsilon}(0)\left(\varepsilon-\varepsilon_{*}\right)<0$ then $u=M$ is the unique solution in $M+\mathcal{N}$.

- Under the assumptions (5.1)-(5.3), it results from the proof above that if we parameterize the bifurcating branch along $\operatorname{ker} L\left(\cdot, \varepsilon_{*}\right)=\left\langle\varphi_{*}\right\rangle$ then each point $(u, \varepsilon)$ on this branch has the following representation

$$
u=u(y)=M+v(y)=M+y\left(\varphi_{*}+v_{2}(y, \varepsilon(y))\right) \quad \text { for } y \sim 0 .
$$

Next, some properties of the bifurcation branches will be investigated. First we will describe the shape of these branches near bifurcation points. The usual classification in super/sub critical bifurcation is not well suitable here since it depends on the choice of the parameter. For example, if $\ddot{\varepsilon}(0)>0$ then according to Theorem 5.2,

$$
u=u(\varepsilon) \simeq M+\sqrt{\frac{2}{\tilde{\varepsilon}(0)}\left(\varepsilon-\varepsilon_{*}\right)} \varphi_{*}, \quad \text { for } \varepsilon \sim \varepsilon_{*}, \varepsilon>\varepsilon_{*},
$$

and the bifurcation is supercritical. On the other hand, $L:=1 / \sqrt{\varepsilon}$ is also a convenient parameter and is used as a bifurcation parameter in Section 6. Setting $L_{*}=1 / \sqrt{\varepsilon_{*}}$, we have

$$
u(\varepsilon)=u(L) \simeq M+\sqrt{\frac{2}{\ddot{\varepsilon}(0)} \frac{L_{*}+L}{L^{2} L_{*}^{2}}\left(L_{*}-L\right)} \varphi_{*}, \quad \text { for } L \sim L_{*}, L<L_{*},
$$


and the same bifurcation is now subcritical. Notice that the function $w$ defined by

$$
w(y):=u(\sqrt{\varepsilon} y) \quad \forall y \in(0, L),
$$

is solution to

$$
w^{(4)}+2 w^{(2)}+f(w)=\frac{1}{L} \int_{0}^{L} f(w) \mathrm{d} y \quad \text { in }(0, L) .
$$

Then, in this equation, the parameter $L=1 / \sqrt{\varepsilon}$ is the length $L$ of the domain. For these reasons, and in agreement with the figures of Section 6 , we will use the following definition.

Definition 5.1. Under the assumptions of Theorem 5.2, let

$$
\varepsilon_{k_{*}}:=\frac{1+\sqrt{p}}{\lambda_{k_{*}}}, \quad \bar{\varepsilon}_{k_{*}}:=\frac{1-\sqrt{p}}{\lambda_{k_{*}}} .
$$

Then we will say that the bifurcation branch given by (5.25) is convex at $\left(0, \varepsilon_{*}\right)$ if

$$
\ddot{\varepsilon}(0)\left(\frac{\varepsilon_{k_{*}}+\bar{\varepsilon}_{k_{*}}}{2}-\varepsilon_{*}\right)>0 .
$$

If this quantity is negative, the branch is concave at $\left(0, \varepsilon_{*}\right)$.

Remark 5.2. If the branch is convex at $\varepsilon_{*}=\varepsilon_{k_{*}}$ then it remains locally in the half space $V_{0} \times\left(-\infty, \varepsilon_{k_{*}}\right)$ containing the conjugate bifurcation point $\left(0, \bar{\varepsilon}_{k_{*}}\right)$.

The number

$$
p_{0}:=\left(\frac{-4+\sqrt{10 M^{2}+1}}{5}\right)^{2}
$$

will be useful in the next result. Note that if $M^{2}<3 / 2$, then $0<p_{0}=p_{0}(M) \leq 9 / 25$ with $p_{0}(M)=9 / 25$ if and only if $M=0$.

Theorem 5.3. Under the assumptions of Theorem 5.2, the concavity at $\left(0, \varepsilon_{*}\right)$ of the bifurcating branch is as follows.

- If $\varepsilon_{*}=\varepsilon_{k_{*}}$ then

- if $M^{2} \leq 3 / 2$ then it is convex;

- if $M^{2}>3 / 2$ then

- if $p<p_{0}$ then the branch is concave;

- if $p>p_{0}$ then it is convex.

- If $0<p<1$ and $\varepsilon_{*}=\bar{\varepsilon}_{k_{*}}$ then

- if $M^{2} \geq 3 / 2$ then

- if $p<9 / 25$ then the branch is concave;

- if $p>9 / 25$ then it is convex;

- if $M^{2}<3 / 2$ then

- if $p \in\left(p_{0}, 9 / 25\right)$ then the branch is concave;

- if $p \notin\left[p_{0}, 9 / 25\right]$ then it is convex.

Notice that the convexity or concavity at $\left(0, \varepsilon_{*}\right)$ does not depend on $k_{*}$.

Proof. By Definition 5.1, the proof consists essentially in computing the sign of $\ddot{\varepsilon}(0)$. By (5.23), it is equal to

$$
\ddot{\varepsilon}(0)=\frac{\varepsilon_{*} / 8}{\varepsilon_{*} \lambda_{*}-f^{\prime}(M)} \frac{f^{\prime \prime}(M)^{2}-f^{\prime \prime \prime}(M) P\left(\varepsilon_{*} \lambda_{2 k_{*}}\right)}{P\left(\varepsilon_{*} \lambda_{2 k_{*}}\right)},
$$


where $P(X):=(X-1)^{2}-p$, for every $X \in \mathbb{R}$. We will now find the sign of these numbers.

Sign of $\varepsilon_{*} \boldsymbol{\lambda}_{*}-\boldsymbol{f}^{\prime}(\boldsymbol{M})$. If $\varepsilon_{*}=\varepsilon_{k_{*}}$ then $\varepsilon_{*} \lambda_{*}-f^{\prime}(M)=p+\sqrt{p}$ is positive. If $p<1$ and $\varepsilon_{*}=\bar{\varepsilon}_{k_{*}}$ then $\varepsilon_{*} \lambda_{*}-f^{\prime}(M)=p-\sqrt{p}$ is negative.

Sign of $\boldsymbol{P}\left(\varepsilon_{*} \boldsymbol{\lambda}_{\mathbf{2} \boldsymbol{k}_{*}}\right)$. If $\varepsilon_{*}=\varepsilon_{k_{*}}$ then $\varepsilon \mapsto P\left(\varepsilon \lambda_{2 k_{*}}\right)$ has two roots $\varepsilon_{2 k_{*}}$ and $\bar{\varepsilon}_{2 k_{*}}$. Since $\bar{\varepsilon}_{2 k_{*}}<\varepsilon_{2 k_{*}}<\varepsilon_{*}$, it results that $P\left(\varepsilon_{*} \lambda_{2 k_{*}}\right)>0$. If $p<1$ and $\varepsilon_{*}=\bar{\varepsilon}_{k_{*}}$ then $\bar{\varepsilon}_{2 k_{*}}<\varepsilon_{*}$. Moreover

$$
\varepsilon_{*}-\varepsilon_{2 k_{*}}=\frac{3-5 \sqrt{p}}{4 \lambda_{k_{*}}} .
$$

Thus $P\left(\varepsilon_{*} \lambda_{2 k_{*}}\right)>0$ if and only if $p<9 / 25$. It should be noticed that $p=9 / 25$ yields that $P\left(\varepsilon_{*} \lambda_{2 k_{*}}\right)=0$ and $\operatorname{ker} L\left(\cdot, \varepsilon_{*}\right)$ is generated by $\varphi_{k_{*}}$ and $\varphi_{2 k_{*}}$. However, this kernel has dimension one by assumption. Hence $p$ cannot be equal to $9 / 25$. With the notation of Proposition 4.1, the case $p=9 / 25$ corresponds to the case $p=p_{2 k_{*}, k_{*}}$.

Sign of $A:=f^{\prime \prime}(M)^{2}-f^{\prime \prime \prime}(M) P\left(\varepsilon_{*} \lambda_{2 k_{*}}\right)$.

Since $P\left(\varepsilon_{*} \lambda_{2 k_{*}}\right)=3\left(8 \varepsilon_{*} \lambda_{*}-5 f^{\prime}(M)\right)$ and $f^{\prime \prime}(M)=6 M$, there holds

$$
A / 18=2 M^{2}-8 \varepsilon_{*} \lambda_{*}+5 f^{\prime}(M) .
$$

If $\varepsilon_{*}=\varepsilon_{k_{*}}$ then $A / 18=-5 p-8 \sqrt{p}+2 M^{2}-3$. Hence, we can find easily the sign of $A$ by observing that $A=0$ if $p=p_{0}$ and $M^{2}>3 / 2$. If $M^{2} \leq 3 / 2$ then $A<0$ for every positive $p$. If $\varepsilon_{*}=\bar{\varepsilon}_{k_{*}}$ then $A / 18=-5 p+8 \sqrt{p}+2 M^{2}-3$. The sign of $A$ is determinated as in the previous case.

The assertions of the theorem follow from the above analysis.

Principle of Exchange of Stability. It expresses that when the trivial solution loose its stability, then the bifurcating branch recovers the stability lost by the trivial solution. More precisely, in accordance with [Kie04], we give the following definition.

Definition 5.2. Let $\mathcal{S} \subset \dot{V}_{0} \times(0, \infty)$ be a branch of solutions to (2.14) bifurcating from $\left(0, \varepsilon_{*}\right)$. We say that $\mathcal{S}$ satisfies the Principle of Exchange of Stability at $\left(0, \varepsilon_{*}\right)$ if

- the trivial branch $(0,(0, \infty))$ changes its stability at $\left(0, \varepsilon_{*}\right)$, i.e. from stable for $\varepsilon<\varepsilon_{*}$ becomes unstable for $\varepsilon>\varepsilon_{*}$, or conversely;

- for every $(v, \varepsilon) \in \mathcal{S}$ with $v \neq 0,(v, \varepsilon)$ and $(0, \varepsilon)$ have opposite stability.

The Principle of Exchange of Stability is illustrated in Figure 2 for a supercritical bifurcation. In order to prove that this principles holds for $(2.14)$, we first need a parameterization of the eigenmodes. We recall that $\varphi_{*}^{\perp}$ is given by (5.8) and that

$$
P(X):=(X-1)^{2}-p, \quad \forall X \in \mathbb{R} .
$$

Proposition 5.4. Under the assumptions of Theorem 5.2, there exist $\delta>0$, and smooth functions $\mu:(-\delta, \delta) \rightarrow \mathbb{R}$ and $w_{2}:(-\delta, \delta) \rightarrow \varphi_{*}^{\perp}$ such that $\mu(0)=0, w_{2}(0)=0$ and

$$
D_{v} F(v(y), \varepsilon(y))\left(\varphi_{*}+w_{2}(y)\right)=\mu(y)\left(\varphi_{*}+w_{2}(y)\right) \quad \forall y \in(-\delta, \delta)
$$

where $F$ is defined by (2.13) and $v(\cdot)$ by (5.26). Moreover, $\dot{\mu}(0)=0$ and (see (5.17), Lemma 5.1)

$$
\ddot{\mu}(0)=2 \partial_{y}^{2} g\left(0, \varepsilon_{*}\right)=-\frac{f^{\prime \prime}(M)^{2}-f^{\prime \prime \prime}(M) P\left(\varepsilon_{*} \lambda_{2 k_{*}}\right)}{2 P\left(\varepsilon_{*} \lambda_{2 k_{*}}\right)} .
$$

In (5.29), $\{v(y), \varepsilon(y) \mid y \in(-\delta, \delta)\}$ is the bifurcating branch and $\left(\varphi_{*}+w_{2}(\cdot), \mu(\cdot)\right)$ is a one parameter family of eigenmodes. 


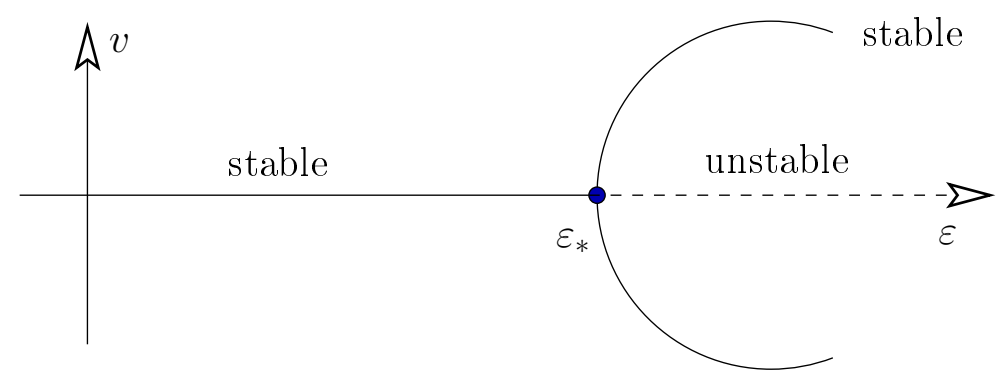

Figure 2. Principle of Exchange of Stability

Proof. The existence of smooth functions $\mu(\cdot)$ and $w_{2}(\cdot)$ satisfying (5.29) follows from [Kie04, Proposition I.7.2] . Hence there remains to compute the derivative of $\mu$. For this, since $w_{2}(y)$ belongs to $\varphi_{*}^{\perp}$, the function

$$
A(y):=P D_{v} F(v(y), \varepsilon(y))\left(\varphi_{*}+w_{2}(y)\right)
$$

satisfies $A(y)=\mu(y) \varphi_{*}$ by (5.29). Moreover, recalling (5.13), we have

$$
y D N(y v)=y^{2} D_{v} \bar{N}(v, y) .
$$

Thus, by differentiating (5.29) w.r.t. $y$,

$$
\begin{aligned}
\dot{A}(y)=P L\left(\dot{w}_{2}, \varepsilon\right)+P \partial_{\varepsilon} L\left(\varphi_{*}+w_{2}, \varepsilon\right) \dot{\varepsilon} & +P D_{v} \bar{N}\left(\varphi_{*}+v_{2}, y\right)\left(\varphi_{*}+w_{2}\right) \\
& +y P \partial_{y}\left\{D_{v} \bar{N}\left(\varphi_{*}+v_{2}, y\right)\left(\varphi_{*}+w_{2}\right)\right\} .
\end{aligned}
$$

Since $P L\left(\cdot, \varepsilon_{*}\right)=0, \dot{\varepsilon}(0)=0$ (see $\left.(5.22)\right)$ and $v_{2}\left(0, \varepsilon_{*}\right)=w_{2}(0)=0$,

$$
\dot{A}(0)=P D_{v} \bar{N}\left(\varphi_{*}, 0\right) \varphi_{*}=P\left(f^{\prime \prime}(M)\left(\varphi_{*}^{2}-\int_{\Omega} \varphi_{*}^{2}\right)\right)=0,
$$

according to (5.6). Thus, $\dot{\mu}(0)=0$. Differentiating (5.30), we obtain

$$
\ddot{A}(0)=P \partial_{\varepsilon} L\left(\varphi_{*}, \varepsilon_{*}\right) \ddot{\varepsilon}(0)+2 P \partial_{y}\left\{D_{v} \bar{N}\left(\varphi_{*}+v_{2}, y\right)\left(\varphi_{*}+w_{2}\right)\right\}_{\mid y=0} .
$$

This latter term is equal to

$$
4 f^{\prime \prime}(M) \int_{\Omega} \varphi_{*}^{2} \partial_{y}\left\{v_{2}(y, \varepsilon(y))\right\}_{\mid y=0} \mathrm{~d} x+4 f^{\prime \prime}(M) \int_{\Omega} \varphi_{*}^{2} \dot{w}(0)+2 f^{\prime \prime \prime}(M) \int_{\Omega} \varphi_{*}^{4} \mathrm{~d} x .
$$

Moreover since $\dot{\varepsilon}(0)=0$,

$$
\partial_{y}\left\{v_{2}(y, \varepsilon(y))\right\}_{\mid y=0}=\partial_{y} v_{2}\left(0, \varepsilon_{*}\right) .
$$

Thus the first integral is given by (5.20). For the second integral, we observe that $\dot{w}(0)=2 \partial_{y} v_{2}\left(0, \varepsilon_{*}\right)$. Indeed, arguing as in (5.30) but projecting instead on $\varphi_{*}^{\perp}$, we obtain

$$
L\left(\dot{w}(0), \varepsilon_{*}\right)+f^{\prime \prime}(M)\left(\varphi_{*}^{2}-\int_{\Omega} \varphi_{*}^{2}\right)=0 .
$$

the claim follows by comparison with (5.15). 
Then, in view of Lemma 5.1,

$$
\begin{aligned}
2 P \partial_{y}\left\{D_{v} \bar{N}\left(\varphi_{*}+v_{2}, y\right)\right. & \left.\left(\varphi_{*}+w_{2}\right)\right\}\left.\right|_{y=0} \\
& =3\left(4 f^{\prime \prime}(M) \int_{\Omega} \varphi_{*}^{2} \partial_{y} v_{2}\left(0, \varepsilon_{*}\right) \mathrm{d} x+\frac{2}{3} f^{\prime \prime \prime}(M) \int_{\Omega} \varphi_{*}^{4} \mathrm{~d} x\right) \\
& =3 \partial_{y}^{2} g\left(0, \varepsilon_{*}\right) .
\end{aligned}
$$

Since $P \partial_{\varepsilon} L\left(\varphi_{*}, \varepsilon_{*}\right)=P\left(2 \varepsilon_{*} \varphi_{*}^{(4)}+2 \varphi_{*}^{(2)}\right)=\partial_{\varepsilon} g\left(0, \varepsilon_{*}\right)$, we obtain with (5.31), (5.33) and (5.23),

$$
\ddot{A}(0)=2 \partial_{y}^{2} g\left(0, \varepsilon_{*}\right) .
$$

The value of $\partial_{y}^{2} g\left(0, \varepsilon_{*}\right)$ is given by Lemma 5.1 . We conclude by noticing that $\ddot{\mu}(0) \varphi_{*}=$ $\ddot{A}(0)$.

Theorem 5.5. Let us assume (5.1)-(5.3) and

$$
P\left(\varepsilon_{*} \lambda_{2 k_{*}}\right) \neq f^{\prime \prime 2} / f^{\prime \prime \prime}(M) .
$$

If the trivial branch changes its stability at $\left(0, \varepsilon_{*}\right)$ then the Principle of Exchange of Stability holds at $\left(0, \varepsilon_{*}\right)$ for Equation (2.14).

Recall that Proposition 4.2 gives necessary and sufficient conditions for the trivial branch to change its stability at $\left(0, \varepsilon_{*}\right)$.

Proof. By assumption $v=0$ changes its stability at $\left(0, \varepsilon_{*}\right)$. With (5.3), we deduce that all the eigenvalues of $L\left(\cdot, \varepsilon_{*}\right)$ are positive except $\left(\varepsilon_{*} \lambda_{k_{*}}-1\right)^{2}-p$, which is zero. By continuity of the eigenvalues, the stability of the bifurcating branch will be determinated by the sign of the function $\mu$ given in Proposition 5.4. Since $\dot{\mu}(0)=0$,

$$
\mu(y)=\frac{1}{2} \ddot{\mu}(0) y^{2}+O\left(y^{3}\right) .
$$

By (5.34) and Proposition 5.4, $\ddot{\mu}(0) \neq 0$. Thus its sign determinates the stability of the bifurcation branch. Since $v=0$ changes its stability at $\left(0, \varepsilon_{*}\right), \varepsilon \mapsto P\left(\varepsilon \lambda_{*}\right)$ changes its sign at $\varepsilon=\varepsilon_{*}$. Thus, we must have $P^{\prime}\left(\varepsilon_{*} \lambda_{k_{*}}\right)=2\left(\varepsilon_{*} \lambda_{k_{*}}-1\right) \neq 0$.

- If it is positive then $v=0$ is unstable for $\varepsilon<\varepsilon_{*}$ and stable if $\varepsilon>\varepsilon_{*}$. Moreover, $\varepsilon_{*}=\varepsilon_{k_{*}}$ hence $\varepsilon_{*} \lambda_{k_{*}}-f^{\prime}(M)>0$ since $p>0$. Thus by (5.23), (5.19) and Proposition 5.4 ,

$$
\operatorname{sgn} \ddot{\varepsilon}(0)=-\operatorname{sgn} \partial_{y}^{2} g\left(0, \varepsilon_{*}\right)=-\operatorname{sgn} \ddot{\mu}(0) .
$$

If $\ddot{\varepsilon}(0)>0$ then $\ddot{\mu}(0)<0, \varepsilon=\varepsilon(y)>\varepsilon_{*}$ and the branch is unstable. Hence the Principle of Exchange of Stability holds in this case. We check also in the same way that it remains true for $\ddot{\varepsilon}(0)<0$.

- If $P^{\prime}\left(\varepsilon_{*} \lambda_{k_{*}}\right)=2\left(\varepsilon_{*} \lambda_{k_{*}}-1\right)<0$ then $v=0$ is stable for $\varepsilon<\varepsilon_{*}$ and unstable if $\varepsilon>\varepsilon_{*}$. In that case, we must have $\varepsilon_{*}=\bar{\varepsilon}_{k_{*}}$. Consequently, $p \in(0,1)$ and

$$
\varepsilon_{*} \lambda_{k_{*}}-f^{\prime}(M)=p-\sqrt{p}<0 .
$$

Thus $\ddot{\varepsilon}(0)$ and $\ddot{\mu}(0)$ possess the same sign. If $\ddot{\mu}(0)>(<) 0$ then the bifurcating branch is stable (resp. unstable) for $\varepsilon>(<) \varepsilon_{*}$ and the Principle of Exchange of Stability holds. This completes the proof of the Theorem. 
Comparison between the energy of the trivial and bifurcating solutions. In many situation, the stability of the trivial solution does not change at the bifurcation point $\left(0, \varepsilon_{*}\right)$. For example, Proposition 4.2 shows that $v=0$ does not change its stability if $\varepsilon \in\left(0, \varepsilon_{k_{+}}\right)$. Thus, in these cases, it can be interesting to compare the energy of the trivial solution $(0, \varepsilon)$ with the energy of the non trivial solutions obtained by bifurcation theory. This is done in the theorem below by using the notion of convexity of a bifurcation branch introduced in Definition 5.1. We recall that the energy of a solution $(v, \varepsilon)$ to $(2.14)$ is $E(M+v, \varepsilon)$ where $E$ is defined by (2.9).

Theorem 5.6. Assume that (5.1)-(5.3) and (5.24) hold. If the bifurcation branch of solutions to $(2.14)$ is convex at $\left(0, \varepsilon_{*}\right)$ then $\left(0, \varepsilon_{*}\right)$ is a local maximizer of the energy along this branch. If it is concave at $\left(0, \varepsilon_{*}\right)$ then $\left(0, \varepsilon_{*}\right)$ is a local minimizer the energy along the branch.

Proof. By using the representation (5.26) of the bifurcation branch and noticing that

$$
D_{u} E(u(y), \varepsilon(y))=0 \quad \text { in } \dot{L}^{2}(\Omega),
$$

we have

$$
\begin{aligned}
\frac{\mathrm{d}}{\mathrm{d} y} E(u(y), \varepsilon(y)) & =D_{\varepsilon} E(u(y), \varepsilon(y)) \dot{\varepsilon}(y) \\
& =\dot{\varepsilon} / \varepsilon(y) \int_{\Omega} \varepsilon^{2} u_{x x}^{2}-\varepsilon u_{x}^{2} \mathrm{~d} x,
\end{aligned}
$$

where $\dot{\varepsilon}$ denotes the derivative w.r.t. $y$. If we denote by $A(y)$ the above integral then

$$
\dot{A}(y)=\int_{\Omega} 2 \dot{\varepsilon} \varepsilon u_{x x}^{2}+2 \varepsilon^{2} \dot{u}_{x x} u_{x x}-\dot{\varepsilon} u_{x}^{2}-2 \varepsilon \dot{u}_{x} u_{x} \mathrm{~d} x .
$$

Since $u(0)=M, \dot{u}(0)=\varphi_{*}$ and $\dot{\varepsilon}(0)=0$ by $(5.22)$, we obtain $\dot{A}(0)=0$. Moreover,

$$
\begin{aligned}
\ddot{A}(0) & =\int_{\Omega} \varepsilon_{*}^{2} \dot{u}_{x x}^{2}(0)-2 \varepsilon_{*} \dot{u}_{x}^{2}(0) \mathrm{d} x \\
& =\int_{\Omega} 2 \varepsilon_{*}^{2} \lambda_{*}^{2} \varphi_{*}^{2}-2 \varepsilon_{*} \lambda_{*} \varphi_{*}^{2} \mathrm{~d} x \\
& =\varepsilon_{*}^{2} \lambda_{*}^{2}-\varepsilon_{*} \lambda_{*} .
\end{aligned}
$$

Since $\varepsilon_{*}^{2} \lambda_{*}^{2}-2 \varepsilon_{*} \lambda_{*}+f^{\prime}(M)=0$, we have

$$
\ddot{A}(0)=\varepsilon_{*} \lambda_{*}-f^{\prime}(M)= \begin{cases}p+\sqrt{p}>0 & \text { if } \varepsilon_{*}=\varepsilon_{k_{*}} \\ p-\sqrt{p}<0 & \text { if } 0<p<1 \quad \text { and } \varepsilon_{*}=\bar{\varepsilon}_{k_{*}} .\end{cases}
$$

From the above computations, we deduce that

$$
\frac{\mathrm{d}}{\mathrm{d} y} E(u(y), \varepsilon(y))=\frac{\ddot{\varepsilon} \ddot{A}(0)}{2 \varepsilon_{*}} y^{3}+O\left(y^{4}\right) .
$$

Hence, for $y$ close to 0 ,

$$
\operatorname{sgn} \frac{\mathrm{d}}{\mathrm{d} y} E(u(y), \varepsilon(y))=\left\{\begin{array}{ll}
\operatorname{sgn} \ddot{\varepsilon}(0) y & \text { if } \varepsilon_{*}=\varepsilon_{k_{*}} \\
-\operatorname{sgn} \ddot{\varepsilon}(0) y & \text { if } 0<p<1 \quad \text { and } \varepsilon_{*}=\bar{\varepsilon}_{k_{*}} .
\end{array} .\right.
$$

If the branch is convex at $\left(0, \varepsilon_{*}\right)$ then $\ddot{\varepsilon}(0)\left(\frac{\varepsilon_{k_{*}}+\bar{\varepsilon}_{k_{*}}}{2}-\varepsilon_{*}\right)>0$. 
- If $\varepsilon_{*}=\varepsilon_{k_{*}}$ then $\ddot{\varepsilon}(0)<0$ and

$$
\operatorname{sgn} \frac{\mathrm{d}}{\mathrm{d} y} E(u(y), \varepsilon(y))=-\operatorname{sgn} y
$$

- In the other case, $\ddot{\varepsilon}(0)>0$ and (5.35) still holds.

Thus $\left(0, \varepsilon_{*}\right)$ is a local maximizer of the energy. The concave situation is treated analogously and is left to the reader. This completes the proof of the theorem.

5.1. The case $\boldsymbol{p}=\mathbf{0}$. According to Proposition 4.1, bifurcation occurs at $\left(0, \varepsilon_{*}\right)$ only if there exists a positive integer $k_{*}$ such that

$$
\varepsilon_{*}:=\frac{1}{\lambda_{k_{*}}}=\frac{1}{\lambda_{*}} .
$$

This case is critical since $f^{\prime}(M)=1$ and $\partial_{\varepsilon} g\left(0, \varepsilon_{*}\right)=0$ according to (5.19) hence (5.23) does not make sense. Besides, $\varepsilon_{*}$ is a root of

$$
\varepsilon \mapsto\left(\varepsilon \lambda_{*}-1\right)^{2}-p
$$

with multiplicity two. Nevertheless, (5.3) remains true (by Proposition 4.1) and by using Newton polygon's method (see for instance [Kie04, Section I-15] ), we can prove the following result.

Theorem 5.7. Let us assume that $p=0,(5.36)$ holds and

$$
f^{\prime \prime}(M)^{2}-9 f^{\prime \prime \prime}(M)>0
$$

then $\left(0, \varepsilon_{*}\right)$ is a bifurcation point for Equation (2.14) and there exist locally two distinct branches of solutions through $\left(0, \varepsilon_{*}\right)$, namely $\left(v_{+}(\cdot), \cdot\right)$ and $\left(v_{-}(\cdot), \cdot\right)$. Besides,

$$
v_{ \pm}(\varepsilon)= \pm \frac{\varepsilon-\varepsilon_{*}}{\tilde{\varepsilon}_{0}} \varphi_{*}+O\left(\left(\varepsilon-\varepsilon_{*}\right)^{2}\right) \quad \text { in } \dot{V}_{0},
$$

where $\tilde{\varepsilon}_{0}$ is given by (5.40) below.

Proof. The projection on $R\left(L\left(\cdot, \varepsilon_{*}\right)\right)$ still gives the local solution (5.12) to (5.10). However, since $f^{\prime}(M)=1-p=1$, we have $\partial_{\varepsilon} g\left(0, \varepsilon_{*}\right)=0$ by (5.19). Standard computations then give

$$
\begin{aligned}
\partial_{\varepsilon}^{2} g\left(0, \varepsilon_{*}\right) & =2 P\left(\varphi_{*}^{(4)}\right)=2 \lambda_{*}^{2} \varphi_{*} \neq 0 \\
\partial_{y \varepsilon} g\left(0, \varepsilon_{*}\right) & =0 .
\end{aligned}
$$

Hence

$$
g(y, \varepsilon)=\frac{1}{2} \partial_{y}^{2} g\left(0, \varepsilon_{*}\right) y^{2}+\frac{1}{2} \partial_{\varepsilon}^{2} g\left(0, \varepsilon_{*}\right)\left(\varepsilon-\varepsilon_{*}\right)^{2}+\text { h.o.t. }
$$

According to Newton's polygon method, we put $\varepsilon=\varepsilon_{*}+y \tilde{\varepsilon}$, where $\tilde{\varepsilon} \in \mathbb{R}$ is a new parameter. Then

$$
g\left(y, \varepsilon_{*}+y \tilde{\varepsilon}\right)=\frac{1}{2} y^{2}\left(\partial_{y}^{2} g\left(0, \varepsilon_{*}\right)+\partial_{\varepsilon}^{2} g\left(0, \varepsilon_{*}\right) \tilde{\varepsilon}^{2}+R(y, \tilde{\varepsilon})\right),
$$

where $R: \mathbb{R} \rightarrow\left\langle\varphi_{*}\right\rangle$ satisfies $R(0, \cdot)=0$ and $\partial_{\tilde{\varepsilon}} R(0, \cdot)=0$ since $g$ is analytic and $R$ contains higher order terms according to Newton's polygon method. By Lemma 5.1 and (5.36),

$$
\partial_{y}^{2} g\left(0, \varepsilon_{*}\right)=\frac{1}{4}\left(\frac{-f^{\prime \prime}(M)^{2}}{\left(\varepsilon_{*} \lambda_{2 k_{*}}-1\right)^{2}}+f^{\prime \prime \prime}(M)\right)=\frac{1}{36}\left(-f^{\prime \prime}(M)^{2}+9 f^{\prime \prime \prime}(M)\right) .
$$

Thus, from $(5.37),(5.38)$, there exists $\tilde{\varepsilon}_{0}>0$ satisfying

$$
\partial_{y}^{2} g\left(0, \varepsilon_{*}\right)+\partial_{\varepsilon}^{2} g\left(0, \varepsilon_{*}\right) \tilde{\varepsilon}_{0}^{2}=0 .
$$


That is to say

$$
\tilde{\varepsilon}_{0}=\frac{1}{6}\left(\frac{f^{\prime \prime}(M)^{2}-9 f^{\prime \prime \prime}(M)}{2 \lambda_{*}^{2}}\right)^{1 / 2} .
$$

Moreover, since $\partial_{\tilde{\varepsilon}} R\left(0, \tilde{\varepsilon}_{0}\right)=0$ and $2 \partial_{\varepsilon}^{2} g\left(0, \varepsilon_{*}\right) \tilde{\varepsilon}_{0} \neq 0$, the Implicit Function Theorem yields the existence of a positive number $\delta$ and some functions $\tilde{\varepsilon}_{+}, \tilde{\varepsilon}_{-}:(-\delta, \delta) \rightarrow \mathbb{R}$ such that $\tilde{\varepsilon}_{+}(0)=\tilde{\varepsilon}_{0}, \tilde{\varepsilon}_{-}(0)=-\tilde{\varepsilon}_{0}$ and

$$
\partial_{y}^{2} g\left(0, \varepsilon_{*}\right)+\partial_{\varepsilon}^{2} g\left(0, \varepsilon_{*}\right) \tilde{\varepsilon}_{ \pm}(y)^{2}+R\left(y, \tilde{\varepsilon}_{ \pm}(y)\right)=0, \quad \forall y \in(-\delta, \delta) .
$$

If we put $\varepsilon_{ \pm}(y):=\varepsilon_{*}+y \tilde{\varepsilon}_{ \pm}(y)=\varepsilon_{*} \pm y \tilde{\varepsilon}_{0}+O\left(y^{2}\right)$ then, in view of (5.39),

$$
v_{ \pm}(y):=y\left(\varphi_{*}+v_{2}\left(y, \varepsilon_{ \pm}(y)\right)\right)
$$

give branches of solutions. Going back to the original parameter $\varepsilon$, we get

$$
v=v_{ \pm}(\varepsilon)= \pm \frac{\varepsilon-\varepsilon_{*}}{\tilde{\varepsilon}_{0}} \varphi_{*}+O\left(\left(\varepsilon-\varepsilon_{*}\right)^{2}\right), \quad \text { for } \varepsilon \sim \varepsilon_{*} .
$$

Moreover, since $v_{2}\left(y, \varepsilon_{ \pm}(y)\right) \in \varphi_{*}^{\perp}$, we have

$$
\left(v_{ \pm}(\varepsilon), \varphi_{*}\right)_{2}= \pm \frac{\varepsilon-\varepsilon_{*}}{2 \tilde{\varepsilon}_{0}} \quad \text { for } \varepsilon \sim \varepsilon_{*},
$$

hence $u_{+}(\varepsilon) \neq u_{-}(\varepsilon)$ if $\varepsilon$ is close enough to $\varepsilon_{*}$. This completes the proof of the theorem.

5.2. Symmetries. We will prove that bifurcating solutions obtained by Theorem 5.2 heritate some symmetry properties from $\varphi_{*}$. We first show that these solutions are $2 / k_{*^{-}}$ periodic functions, if they are correctly extend to $\mathbb{R}$. For this purpose, for any $u$ in $L^{2}(\Omega)$, we define $T(u) \in L_{l o c}^{2}(\mathbb{R})$ as the periodic function with period 2 such that $T(u)=u$ in $\Omega$ and

$$
T(u)(x)=u(2-x) \quad \text { for a.e. } x \in[1,2] .
$$

Note that $T\left(\varphi_{k}\right)=\cos (k \pi \cdot)$ for any $k \geq 1$.

Proposition 5.8. Under the assumptions (5.1)-(5.3), there exists a neighbourhood $\mathcal{N}_{1} \times$ $I_{1}$ of $\left(0, \varepsilon_{*}\right)$ in $\dot{V}_{0} \times \mathbb{R}$ such that if $(v, \varepsilon) \in \mathcal{N}_{1} \times I_{1}$ is solution to (2.14) then $T(v)$ is a $2 / k_{*}$-periodic function.

Proof. The idea is to restrict the bifurcation analysis to $2 / k_{*}$-periodic functions. For every positive number $\alpha$, we introduce the following spaces

$$
\begin{aligned}
\dot{V}_{0, \alpha} & =\left\{v \in \dot{V}_{0} \mid T(v) \text { is } \alpha \text {-periodic }\right\} \\
\dot{L}_{\alpha}^{2}(\Omega) & =\left\{v \in \dot{L}^{2}(\Omega) \mid T(v) \text { is } \alpha \text {-periodic }\right\} .
\end{aligned}
$$

Let $k_{*}$ be the positive integer satisfying (5.2), (5.3). It is clear that

$$
\left\{\frac{\varphi_{m k_{*}}}{\left\|\varphi_{m k_{*}}\right\|_{2}} \mid m \in \mathbb{N} \backslash\{0\}\right\} \subset \dot{V}_{0,2 / k_{*}}
$$

is a spectral basis in $\dot{L}_{2 / k_{*}}^{2}(\Omega)$ for the operator $L\left(\cdot, \varepsilon_{*}\right)$. Thus the bifurcation analysis of the beginning of this section may be carried out in the space $\dot{L}_{2 / k_{*}}^{2}(\Omega)$. In particular, if $L\left(\cdot, \varepsilon_{*}\right)$ is viewed as an operator from $\dot{V}_{0,2 / k_{*}}$ into $\dot{L}_{2 / k_{*}}^{2}(\Omega)$, we have

$$
\begin{aligned}
\dot{L}_{2 / k_{*}}^{2}(\Omega) & =R\left(L\left(\cdot, \varepsilon_{*}\right)\right) \oplus \operatorname{ker} L\left(\cdot, \varepsilon_{*}\right) \\
\dot{V}_{0,2 / k_{*}} & =\left(R\left(L\left(\cdot, \varepsilon_{*}\right)\right) \cap \dot{V}_{0,2 / k_{*}}\right) \oplus \operatorname{ker} L\left(\cdot, \varepsilon_{*}\right) .
\end{aligned}
$$


Arguing as in the proof of Theorem 5.2, we obtain a branch of solutions in $\dot{V}_{0,2 / k_{*}} \times(0, \infty)$. We conclude by using the local uniqueness of the bifurcation branches.

Proposition 5.9. Under the assumptions (5.1)-(5.3) and (5.24), let B the largest ball of $\dot{V}_{0}$ centered at 0 and contained in $\mathcal{N}_{1}$. Let

$$
\mathcal{S}_{J}=\{(v(y), \varepsilon(y)) \mid y \in J\}
$$

be the bifurcation branch (5.26) included in $B \times I_{1}$, where $J$ is some real interval containing 0 . Then, for every $y \in J,-y$ belongs to $J,(v(-y), \varepsilon(y)) \in \mathcal{S}_{J}$ and

$$
v(-y)=v(y)\left(\cdot+1 / k_{*}\right) \quad \text { in } \Omega \text {. }
$$

In particular, $\left(v(y)\left(\cdot+1 / k_{*}\right), \varepsilon(y)\right)$ is also solution to $(2.14)$.

In $(5.42), v(y)\left(\cdot+1 / k_{*}\right)$ stands for the restriction of $T(v(y))\left(\cdot+1 / k_{*}\right)$ to $\Omega$, where $T$ is defined by (5.41).

Proof. For $y \in J$, we put

$$
w=T(v(y))_{\mid(0,2)}
$$

Then $w \in H^{4}(0,2)$ and

$$
w^{(i)}=\left\{\begin{array}{ll}
\mathbb{I}_{\Omega} T\left(v^{(i)}(y)\right)-\mathbb{I}_{[1,2)} T\left(v^{(i)}(y)\right) & \text { for } i \in\{1,3\} \\
\left.T\left(v(y)^{(i)}\right)\right|_{(0,2)} & \text { for } i \in\{2,4\}
\end{array} .\right.
$$

Then $w_{0}:=w\left(\cdot+1 /\left.k_{*}\right|_{\Omega}\right.$ satisfies

$$
\varepsilon(y)^{2} w_{0}^{(4)}+2 \varepsilon(y) w_{0}^{(2)}+f\left(M+w_{0}\right)=\int_{\Omega} f\left(M+w_{0}\right) \mathrm{d} x \quad \text { in } L^{2}(\Omega) .
$$

Moreover since $L\left(\cdot, \varepsilon_{*}\right)$ is a self-adjoint operator with compact resolvent,

$$
v(y)=\sum_{k \geq 1} x_{k} \varphi_{k} \quad \text { in } L^{2}(\Omega)
$$

and

$$
\sum_{k \geq 1} P\left(\varepsilon_{*} \lambda_{k}\right)^{2} x_{k}^{2}<\infty
$$

where $x_{k}:=2\left(v(y), \varphi_{k}\right)_{2}$. Thus, using also Proposition 5.8,

$$
v(y)=\sum_{m \geq 1} x_{m k_{*}} \varphi_{m k_{*}} \quad \text { in } H^{4}(\Omega)
$$

We will show that $w_{0} \in V_{0}$. For this, since $T$ is a bounded linear operator from $L^{2}(\Omega)$ into $L^{2}(0,2)$, we have

$$
T(v(y))_{(0,2)}=\left.\sum_{m \geq 1} x_{m k_{*}} T\left(\varphi_{m k_{*}}\right)\right|_{(0,2)} \quad \text { in } H^{4}(0,2) .
$$

Thus

$$
\begin{array}{rlrl}
w_{0} & =\sum_{m \geq 1}(-1)^{m} x_{m k_{*}} \varphi_{m k_{*}} & \text { in } H^{4}(\Omega) \\
w_{0}^{(i)}=\sum_{m \geq 1}(-1)^{m} x_{m k_{*}} \varphi_{m k_{*}}^{(i)} & \text { in } H^{4-i}(\Omega), \quad \forall i=1,2,3 .
\end{array}
$$


As a consequence, $w_{0}^{(1)}=w_{0}^{(3)}=0$ on $\partial \Omega$ and $w_{0} \in V_{0}$.

With (5.44), we deduce that $\left(w_{0}, \varepsilon(y)\right)$ is solution to (2.14). Furthermore, $\left\|w_{0}\right\|_{\dot{V}_{0}}=$ $\|v(y)\|_{\dot{V}_{0}}$ thus $\left(w_{0}, \varepsilon(y)\right) \in B \times I_{1}$. Since the solutions to $(2.14)$ in $B \times I_{1}$ belong to $\mathcal{S}_{J}$, $\left(w_{0}, \varepsilon(y)\right) \in \mathcal{S}_{J}$. That is to say, there exists $z \in J$ such that

$$
\left(w_{0}, \varepsilon(y)\right)=(v(z), \varepsilon(z)) \in \mathcal{S}_{J} .
$$

By (5.26), (5.46), we have

$$
P(v(z))=z \varphi_{k_{*}}=-x_{k_{*}} \varphi_{k_{*}}=-P(v(y))=-y \varphi_{k_{*}} .
$$

Thus $z=-y$; then $v(-y)=w_{0}$ satisfies $(5.42)$ and $(v(-y), \varepsilon(y)) \in \mathcal{S}_{J}$ by (5.47). This completes the proof of the proposition.

5.3. Two-dimensional kernel. We will implement the Lyapunov-Schmidt method when the kernel of $L\left(\cdot, \varepsilon_{*}\right)$ has dimension two. According to Proposition 4.1, this is the case if and only if there exist $p \in(0,1), 1 \leq k_{*}<k_{* *}$ and $\varepsilon_{*}>0$ such that

$$
\begin{aligned}
\varepsilon_{*} \lambda_{k_{*}} & :=1+\sqrt{p} \\
\varepsilon_{*} \lambda_{k_{* *}} & :=1-\sqrt{p} .
\end{aligned}
$$

As above we put

$$
\varphi_{*}=\varphi_{k_{*}}, \quad \varphi_{* *}=\varphi_{k_{* *}}, \quad \lambda_{*}=\lambda_{k_{*}}, \quad \lambda_{* *}=\lambda_{k_{* *}},
$$

where $\lambda_{k}=(k \pi)^{2}$ and $\varphi_{k}=\cos (k \pi \cdot)$. Then

$$
\operatorname{ker} L\left(\cdot, \varepsilon_{*}\right)=\left\langle\varphi_{*}, \varphi_{* *}\right\rangle .
$$

Besides, we will assume that

$$
k_{*} \neq 2 k_{* *}, \quad k_{*} \neq 3 k_{* *} .
$$

We define the projection $P, Q$ as in (5.5) but instead of (5.6), we have

$$
P v=2\left(v, \varphi_{*}\right)_{2} \varphi_{*}+2\left(v, \varphi_{* *}\right)_{2} \varphi_{* *}, \quad \forall v \in \dot{L}^{2}(\Omega) .
$$

We decompose every $v \in \dot{V}_{0}$ in a unique way into $v=\varphi+v_{2}$ where $\varphi \in \operatorname{ker} L\left(\cdot, \varepsilon_{*}\right)$ and $v_{2} \in\left\langle\varphi_{*}, \varphi_{* *}\right\rangle^{\perp}$, the orthogonal space of $\left\langle\varphi_{*}, \varphi_{* *}\right\rangle$ in $\dot{V}_{0}$. Then (2.14) is equivalent to

$$
\left\{\begin{array}{l}
\varepsilon>0,\left(\varphi, v_{2}\right) \in \operatorname{ker} L\left(\cdot, \varepsilon_{*}\right) \times\left\langle\varphi_{*}, \varphi_{* *}\right\rangle^{\perp}, \\
L(\varphi, \varepsilon)+L\left(v_{2}, \varepsilon\right)+N\left(\varphi+v_{2}\right)=0 \quad \text { in } \dot{L}^{2}(\Omega) .
\end{array}\right.
$$

Projection on $\boldsymbol{R}\left(\boldsymbol{L}\left(\cdot, \varepsilon_{*}\right)\right)$. Applying $Q$ to (5.53) and using the Implicit Function Theorem, we obtain a smooth function $v_{2}$ defined in some neighbourhood $\mathcal{N}$ of $\left(0, \varepsilon_{*}\right)$ into $R\left(L\left(\cdot, \varepsilon_{*}\right)\right)$ such that

$$
Q L(\varphi, \varepsilon)+Q L\left(v_{2}(\varphi, \varepsilon), \varepsilon\right)+Q N\left(\varphi+v_{2}(\varphi, \varepsilon)\right)=0 \quad \text { in } \dot{L}^{2}(\Omega),
$$

for every $(\varphi, \varepsilon)$ in $\mathcal{N}$. Next in a standard way, we derive

$$
v_{2}\left(0, \varepsilon_{*}\right)=\partial_{\varepsilon} v_{2}\left(0, \varepsilon_{*}\right)=0, \quad \partial_{\varphi} v_{2}\left(0, \varepsilon_{*}\right)=0
$$

For later use, we will compute $\partial_{\varphi}^{2} v_{2}\left(0, \varepsilon_{*}\right)$. 
Proposition 5.10. Under assumptions (5.48)-(5.51), for every $\varphi=\alpha \varphi_{*}+\beta \varphi_{* *}$ with $\alpha$, $\beta$ real, there holds

$$
\partial_{\varphi}^{2} v_{2}\left(0, \varepsilon_{*}\right)(\varphi, \varphi)=x_{2 k_{*}} \varphi_{2 k_{*}}+x_{k_{*}+k_{* *}} \varphi_{k_{*}+k_{* *}}+x_{k_{*}-k_{* *}} \varphi_{k_{*}-k_{* *}}+x_{2 k_{* *}} \varphi_{2 k_{* *}},
$$

where

$$
\begin{aligned}
x_{2 k_{*}}=-f^{\prime \prime}(M) \frac{\alpha^{2}}{2 P\left(\varepsilon_{*} \lambda_{2 k_{*}}\right)} & x_{k_{*}+k_{* *}}=-f^{\prime \prime}(M) \frac{\alpha \beta}{P\left(\varepsilon_{*} \lambda_{k_{*}+k_{* *}}\right)} \\
x_{k_{*}-k_{* *}}=-f^{\prime \prime}(M) \frac{\alpha \beta}{P\left(\varepsilon_{*} \lambda_{k_{*}-k_{* *}}\right)} & x_{2 k_{* *}}=-f^{\prime \prime}(M) \frac{\beta^{2}}{2 P\left(\varepsilon_{*} \lambda_{2 k_{* *}}\right)} .
\end{aligned}
$$

Proof. By differentiating (5.54) twice w.r.t. $\varphi, w:=\partial_{\varphi}^{2} v_{2}\left(0, \varepsilon_{*}\right)$ solves

$$
Q L\left(w, \varepsilon_{*}\right)+Q f^{\prime \prime}(M)\left(\varphi^{2}-\int_{\Omega} \varphi^{2}\right)=0 \quad \text { in } \dot{L}^{2}(\Omega) .
$$

Since $k_{*} \neq 2 k_{* *}$, we check that $\varphi^{2}-\int_{\Omega} \varphi^{2} \in R\left(L\left(\cdot, \varepsilon_{*}\right)\right)$. Thus

$$
L\left(w, \varepsilon_{*}\right)+f^{\prime \prime}(M)\left(\varphi^{2}-\int_{\Omega} \varphi^{2}\right)=0 .
$$

Testing the above equation with $\varphi_{k}$, using $w \in\left\langle\varphi_{*}, \varphi_{* *}\right\rangle^{\perp}$ and

$$
\varphi^{2}-\int_{\Omega} \varphi^{2}=\frac{\alpha^{2}}{2} \varphi_{2 k_{*}}+\alpha \beta \varphi_{k_{*}+k_{* *}}+\alpha \beta \varphi_{k_{*}-k_{* *}}+\frac{\beta^{2}}{2} \varphi_{2 k_{* *}},
$$

we obtain the expansion of $w$.

Projection on $\left\langle\varphi_{*}, \varphi_{* *}\right\rangle$. By setting for $(\varphi, \varepsilon)$ close to $\left(0, \varepsilon_{*}\right)$,

$$
g(\varphi, \varepsilon):=P L(\varphi, \varepsilon)+P L\left(v_{2}(\varphi, \varepsilon), \varepsilon\right)+P N\left(\varphi+v_{2}(\varphi, \varepsilon), y\right),
$$

we are led to solve

$$
g(\varphi, \varepsilon)=0 \quad \text { in }\left\langle\varphi_{*}, \varphi_{* *}\right\rangle
$$

We have

$$
\partial_{\varphi} g(\varphi, \varepsilon)=P L(\cdot, \varepsilon)+P L\left(\partial_{\varphi} v_{2}, \varepsilon\right)+P D_{v} N\left(\varphi+v_{2}\right)\left(I d_{\left\langle\varphi_{*}, \varphi_{* *}\right\rangle}+\partial_{\varphi} v_{2}\right) .
$$

Then with (5.55),

$$
\begin{gathered}
\partial_{\varepsilon} g\left(0, \varepsilon_{*}\right)=\partial_{\varepsilon}^{2} g\left(0, \varepsilon_{*}\right)=0, \quad \partial_{\varphi} g\left(0, \varepsilon_{*}\right)=0 \\
\partial_{\varphi}^{2} g\left(0, \varepsilon_{*}\right)(\varphi, \varphi)=0 \quad \forall \varphi \in\left\langle\varphi_{*}, \varphi_{* *}\right\rangle .
\end{gathered}
$$

Indeed,

$$
\partial_{\varphi}^{2} g(0, \varepsilon)=P L\left(\partial_{\varphi}^{2} v_{2}, \varepsilon\right)+P D_{v}^{2} N\left(\varphi+v_{2}\right)\left[I d_{\left\langle\varphi_{*}, \varphi_{* *}\right\rangle}+\partial_{\varphi} v_{2}\right]^{2}+P D_{v} N\left(\varphi+v_{2}\right) \partial_{\varphi}^{2} v_{2}
$$

Hence

$$
\partial_{\varphi}^{2} g\left(0, \varepsilon_{*}\right)(\varphi, \varphi)=P\left(f^{\prime \prime}(M)\left(\varphi^{2}-\int_{\Omega} \varphi^{2}\right)\right)
$$

Moreover, $\left(\varphi^{2}, \varphi_{*}\right)_{2}=\left(\varphi^{2}, \varphi_{* *}\right)_{2}=0$ since $k_{*} \neq 2 k_{* *}$. Hence (5.58) holds (see (5.52)). By differentiating (5.57) w.r.t. $\varepsilon$, we obtain for all $(\alpha, \beta) \in \mathbb{R}^{2}$,

$$
\partial_{\varphi \varepsilon} g\left(0, \varepsilon_{*}\right)\left(\alpha \varphi_{*}+\beta \varphi_{* *}\right)=2 \alpha \frac{\varepsilon_{*} \lambda_{*}-f^{\prime}(M)}{\varepsilon_{*}} \varphi_{*}+2 \beta \frac{\varepsilon_{*} \lambda_{* *}-f^{\prime}(M)}{\varepsilon_{*}} \varphi_{* *} .
$$


Also

$$
\partial_{\varphi}^{3} g\left(0, \varepsilon_{*}\right)(\varphi, \varphi, \varphi)=P\left(f^{\prime \prime \prime}(M) \varphi_{*}^{3}\right)+3 P\left(f^{\prime \prime}(M) \varphi \partial_{\varphi}^{2} v_{2}\left(0, \varepsilon_{*}\right)(\varphi, \varphi)\right) .
$$

By Proposition 5.10 and $3 k_{* *} \neq k_{*}$, we deduce that

Moreover

$$
\begin{aligned}
3 P\left(f^{\prime \prime}(M) \varphi \partial_{\varphi}^{2} v_{2}\left(0, \varepsilon_{*}\right)(\varphi, \varphi)\right) & =\frac{3}{2} f^{\prime \prime}(M)\left(x_{2 k_{*}} \alpha+\left(x_{k_{*}+k_{* *}}+x_{k_{*}-k_{* *}}\right) \beta\right) \varphi_{*} \\
& +\frac{3}{2} f^{\prime \prime}(M)\left(\left(x_{k_{*}+k_{* *}}+x_{k_{*}-k_{* *}}\right) \alpha+x_{2 k_{* *}} \beta\right) \varphi_{* *} .
\end{aligned}
$$

Thus by (5.60),

$$
P\left(f^{\prime \prime \prime}(M) \varphi^{3}\right)=\frac{3}{2} f^{\prime \prime \prime}(M)\left(\frac{\alpha^{3}}{2}+\alpha \beta^{2}\right) \varphi_{*}+\frac{3}{2} f^{\prime \prime \prime}(M)\left(\frac{\beta^{3}}{2}+\alpha^{2} \beta\right) \varphi_{* *} .
$$

$$
\begin{aligned}
\partial_{\varphi}^{3} g\left(0, \varepsilon_{*}\right)(\varphi, \varphi, \varphi)= \\
\\
\quad \frac{3}{2}\left\{f^{\prime \prime \prime}(M)\left(\frac{\alpha^{3}}{2}+\alpha \beta^{2}\right)+f^{\prime \prime}(M)\left(x_{2 k_{*}} \alpha+\left(x_{k_{*}+k_{* *}}+x_{k_{*}-k_{* *}}\right) \beta\right)\right\} \varphi_{*} \\
+\frac{3}{2}\left\{f^{\prime \prime \prime}(M)\left(\frac{\beta^{3}}{2}+\alpha^{2} \beta\right)+f^{\prime \prime}(M)\left(\left(x_{k_{*}+k_{* *}}+x_{k_{*}-k_{* *}}\right) \alpha+x_{2 k_{* *}} \beta\right)\right\} \varphi_{* *} .
\end{aligned}
$$

The following theorem states a bifurcation result when the kernel of the linearized operator is two-dimensional.

Theorem 5.11. Under assumptions (5.48)-(5.50), $\left(0, \varepsilon_{*}\right)$ is a bifurcation point for Equation (2.14) and there exists a branch $\left(v_{*}(\cdot), \varepsilon(\cdot)\right)$ of non trivial solutions through $\left(0, \varepsilon_{*}\right)$. Besides, if

then these solutions satisfy

$$
P\left(\varepsilon_{*} \lambda_{2 k_{*}}\right) \neq f^{\prime \prime 2} / f^{\prime \prime \prime}(M)
$$

$$
v_{*}(\varepsilon) \simeq \pm \sqrt{\frac{2}{\dot{\varepsilon}_{*}(0)}\left(\varepsilon-\varepsilon_{*}\right)} \varphi_{*} \quad \text { in } \dot{V}_{0},
$$

where

$$
\ddot{\varepsilon}_{*}(0)=\frac{\varepsilon_{*} / 8}{\varepsilon_{*} \lambda_{*}-f^{\prime}(M)}\left(\frac{f^{\prime \prime}(M)^{2}}{P\left(\varepsilon_{*} \lambda_{2 k_{*}}\right)}-f^{\prime \prime \prime}(M)\right)
$$

and $\varepsilon \sim \varepsilon_{*}$ is such that $\ddot{\varepsilon}_{*}(0)\left(\varepsilon-\varepsilon_{*}\right)>0$. Moreover, if $k_{*} \neq 2 k_{* *}, k_{*} \neq 3 k_{* *}$ and

$$
P\left(\varepsilon_{*} \lambda_{2 k_{* *}}\right) \neq f^{\prime \prime 2} / f^{\prime \prime \prime}(M)
$$

then there is another bifurcation branch through $\left(0, \varepsilon_{*}\right)$ satisfying

$$
v_{* *}(\varepsilon) \simeq \pm \sqrt{\frac{2}{\bar{\varepsilon}_{* *}(0)}\left(\varepsilon-\varepsilon_{*}\right)} \varphi_{* *} \quad \text { in } \dot{V}_{0},
$$

where

and $\ddot{\varepsilon}_{* *}(0)\left(\varepsilon-\varepsilon_{*}\right)>0$.

$$
\ddot{\varepsilon}_{* *}(0)=\frac{\varepsilon_{*} / 8}{\varepsilon_{*} \lambda_{* *}-f^{\prime}(M)}\left(\frac{f^{\prime \prime}(M)^{2}}{P\left(\varepsilon_{*} \lambda_{2 k_{* *}}\right)}-f^{\prime \prime \prime}(M)\right)
$$

Remark 5.3. • $\varepsilon_{*} \lambda_{*}-f^{\prime}(M)$ and $\varepsilon_{*} \lambda_{* *}-f^{\prime}(M)$ are non zero due to (5.48), (5.49) and $0<p<1$.

- $P\left(\varepsilon_{*} \lambda_{2 k_{* *}}\right)=0$ if and only if $\varepsilon_{*}=\varepsilon_{2 k_{* *}}$ or $\varepsilon_{*}=\bar{\varepsilon}_{2 k_{* *}}$ (see (4.4)). By (5.49), $\varepsilon_{*}=\bar{\varepsilon}_{k_{* *}} \neq$ $\bar{\varepsilon}_{2 k_{* *}}$. By (5.48), $\varepsilon_{*}=\varepsilon_{k_{*}}$; thus $\varepsilon_{*}=\varepsilon_{2 k_{* *}}$ yields $2 k_{* *}=k_{*}$. It results from (5.51) that $P\left(\varepsilon_{*} \lambda_{2 k_{* *}}\right) \neq 0$ in $(5.64)$.

- The sign of $\ddot{\varepsilon}_{*} \ddot{\varepsilon}_{* *}(0)$ determinates if the branches $\left(v_{*}, \varepsilon\right),\left(v_{* *}, \varepsilon\right)$ are in the same half 
space of $\dot{V}_{0} \times \mathbb{R}$ with boundary $\dot{V}_{0} \times\left\{\varepsilon_{*}\right\}$. From Theorem 5.3, we deduce that they are in the same half space (i.e. $\ddot{\varepsilon}_{*} \ddot{\varepsilon}_{* *}(0)>0$ ) if

$$
\min \left(p_{0}, 9 / 25\right)<p<\max \left(p_{0}, 9 / 25\right) .
$$

Proof of Theorem 5.11. According to the proof of Proposition 5.8 and using the space $\dot{L}_{2 / k_{*}}^{2}(\Omega)$, the bifurcation analysis can be reduced to the case where the kernel of $L\left(\cdot, \varepsilon_{*}\right)$ is $\left\langle\varphi_{k_{*}}\right\rangle$. Thus arguing as in the proof of Theorem 5.2, we obtain the branch $\left(v_{*}, \varepsilon\right)$. If $k_{*}$ is not a multiple of $k_{* *}$ then we work in the space $\dot{L}_{2 / k_{* *}}^{2}(\Omega)$ to get the second branch $\left(v_{* *}, \varepsilon\right)$.

However if $k_{*}=m k_{* *}$ with $m \in \mathbb{N}, m \geq 4$, the dimension of the kernel of $L\left(\cdot, \varepsilon_{*}\right)$ cannot be reduced. Hence we will use the Newton polygon method (see [Kie04, Section I-15]) for Equation (5.56) in order to prove that there is a bifurcation branch tangent to $\varphi_{* *}$. By $(5.58)$

$$
g(\varphi, \varepsilon)=\partial_{\varphi \varepsilon} g\left(0, \varepsilon_{*}\right) \varphi\left(\varepsilon-\varepsilon_{*}\right)+\frac{1}{6} \partial_{\varphi}^{3} g\left(0, \varepsilon_{*}\right)(\varphi, \varphi, \varphi)+\text { h.o.t. }
$$

Let $\tilde{\varepsilon}:=\varepsilon-\varepsilon_{*}$ be positive and $\varphi=\tilde{\varepsilon}^{1 / 2} \tilde{\varphi}$. We have

$$
g\left(\tilde{\varepsilon}^{1 / 2} \tilde{\varphi}, \varepsilon_{*}+\tilde{\varepsilon}\right)=\tilde{\varepsilon}^{3 / 2}\left(\partial_{\varphi \varepsilon} g\left(0, \varepsilon_{*}\right) \tilde{\varphi}+\frac{1}{6} \partial_{\varphi}^{3} g\left(0, \varepsilon_{*}\right)(\tilde{\varphi}, \tilde{\varphi}, \tilde{\varphi})+R(\tilde{\varphi}, \tilde{\varepsilon})\right),
$$

where $R(\cdot, 0)=0$ and $\partial_{\tilde{\varphi}} R(\cdot, 0)=0$ since $g$ is analytic and $R$ contains higher order terms according to Newton's polygon method. By the Implicit Function Theorem,

$$
g\left(\tilde{\varepsilon}^{1 / 2} \tilde{\varphi}, \varepsilon_{*}+\tilde{\varepsilon}\right)=0
$$

has non trivial solutions for $(\tilde{\varphi}, \tilde{\varepsilon})$ close to $\left(\tilde{\varphi}_{0}, 0\right)$ if there exists $\beta \neq 0$ such that the function $\tilde{\varphi}_{0}:=\beta \varphi_{* *}$ satisfies the two conditions:

$$
\begin{gathered}
\partial_{\varphi \varepsilon} g\left(0, \varepsilon_{*}\right) \tilde{\varphi}_{0}+\frac{1}{6} \partial_{\varphi}^{3} g\left(0, \varepsilon_{*}\right)\left(\tilde{\varphi}_{0}, \tilde{\varphi}_{0}, \tilde{\varphi}_{0}\right)=0 \\
\partial_{\varphi \varepsilon} g\left(0, \varepsilon_{*}\right) \cdot+\frac{1}{6} D_{\tilde{\varphi}}\left(\partial_{\varphi}^{3} g\left(0, \varepsilon_{*}\right)(\tilde{\varphi}, \tilde{\varphi}, \tilde{\varphi})\right)_{\left.\right|_{\tilde{\varphi}=\tilde{\varphi}}} \text { is an isomorphism of } \operatorname{ker} L\left(\cdot, \varepsilon_{*}\right) .
\end{gathered}
$$

By (5.59), (5.61), Proposition 5.10 and (5.64), non trivial solutions to (5.65) are given by

$$
\beta^{2}=2 / \ddot{\varepsilon}_{* *}(0) .
$$

Observe that there exists $\beta \in \mathbb{R}$ satisfying (5.67) since $\ddot{\varepsilon}_{* *}(0)>0$. Indeed, $\lambda_{* *}<\lambda_{2 k_{* *}}<$ $\lambda_{*}$ since $m>2$ thus $P\left(\varepsilon_{*} \lambda_{2 k_{* *}}\right)<0$. Moreover $\varepsilon_{*} \lambda_{* *}-f^{\prime}(M)=p-\sqrt{p}<0$ since $p \in(0,1)$. Thus $\ddot{\varepsilon}_{* *}(0)>0$ in view of $(5.64)$.

The matrix of the linear mapping in (5.66) is diagonal with coefficients

$$
\begin{gathered}
2 \frac{\varepsilon_{*} \lambda_{*}-f^{\prime}(M)}{\varepsilon_{*}}+\frac{1}{4}\left(f^{\prime \prime \prime}(M)-f^{\prime \prime}(M)^{2}\left(\frac{1}{P\left(\varepsilon_{*} \lambda_{k_{*}+k_{* *}}\right)}+\frac{1}{P\left(\varepsilon_{*} \lambda_{k_{*}-k_{* *}}\right)}\right)\right) \beta^{2} \\
2 \frac{\varepsilon_{*} \lambda_{* *}-f^{\prime}(M)}{\varepsilon_{*}}+\frac{3}{8}\left(f^{\prime \prime \prime}(M)-\frac{f^{\prime \prime}(M)^{2}}{P\left(\varepsilon_{*} \lambda_{2 k_{* *}}\right)}\right) \beta^{2} .
\end{gathered}
$$

By $(5.64),(5.67)$, this latter entry is equal to $-4\left(\varepsilon_{*} \lambda_{* *}-f^{\prime}(M)\right) / \varepsilon_{*} \neq 0$. Hence if the former entry above is non zero then (5.66) holds. Since $\varepsilon_{*} \lambda_{*}-f^{\prime}(M)$ is positive, it is enough to show that

$$
\frac{1}{P\left(\varepsilon_{*} \lambda_{k_{*}+k_{* *}}\right)}+\frac{1}{P\left(\varepsilon_{*} \lambda_{k_{*}-k_{* *}}\right)}=\frac{P\left(\varepsilon_{*} \lambda_{k_{*}+k_{* *}}\right)+P\left(\varepsilon_{*} \lambda_{k_{*}-k_{* *}}\right)}{P\left(\varepsilon_{*} \lambda_{k_{*}+k_{* *}}\right) P\left(\varepsilon_{*} \lambda_{k_{*}-k_{* *}}\right)}<0
$$


The denominator is negative since $m>2$. Next, we have

$$
\begin{aligned}
P\left(\varepsilon_{*} \lambda_{k_{*}+k_{* *}}\right) & =\left((m+1)^{2} \varepsilon_{*}\left(\pi k_{* *}\right)^{2}-1\right)^{2}-p \\
& =(1-\sqrt{p}) m(m+2)\left(-\left(m^{2}+2 m+2\right) \sqrt{p}+(m+1)^{2}-1\right) .
\end{aligned}
$$

Now,

$$
1+\sqrt{p}=\varepsilon_{*} \lambda_{k_{*}}=m^{2} \varepsilon_{*} \lambda_{k_{* *}}=m^{2}(1-\sqrt{p}),
$$

thus

$$
\sqrt{p}=\frac{m^{2}-1}{m^{2}+1}
$$

Then

$$
P\left(\varepsilon_{*} \lambda_{k_{*}+k_{* *}}\right)=(1-\sqrt{p}) \frac{2 m}{m^{2}+1}(m+2)(2 m+1) .
$$

In the same way, we have

$$
P\left(\varepsilon_{*} \lambda_{k_{*}-k_{* *}}\right)=(1-\sqrt{p}) \frac{2 m}{m^{2}+1}(m-2)(-2 m+1) .
$$

From these two equalities, it results that

$$
P\left(\varepsilon_{*} \lambda_{k_{*}+k_{* *}}\right)+P\left(\varepsilon_{*} \lambda_{k_{*}-k_{* *}}\right)=(1-\sqrt{p}) \frac{20 m^{2}}{m^{2}+1}>0,
$$

and (5.68) follows. Hence (5.66) is proved.

Denoting by $\beta$ the positive solution to (5.67), we deduce from the above analysis that there exists a continuous function $\tilde{\varphi}:[0, \delta) \rightarrow \operatorname{ker} L\left(\cdot, \varepsilon_{*}\right)$ such that $\tilde{\varphi}(0)=\beta \varphi_{* *}$ and

$$
g\left(\tilde{\varepsilon}^{1 / 2} \tilde{\varphi}(\tilde{\varepsilon}), \varepsilon_{*}+\tilde{\varepsilon}\right)=0, \quad \forall \tilde{\varepsilon} \in[0, \delta) .
$$

By setting $\varphi(\varepsilon):=\left(\varepsilon-\varepsilon_{*}\right)^{1 / 2} \tilde{\varphi}\left(\varepsilon-\varepsilon_{*}\right)$ and

$$
v_{* *}(\varepsilon):=\varphi(\varepsilon)+v_{2}(\varphi(\varepsilon), \varepsilon), \quad \forall \varepsilon \in\left[\varepsilon_{*}, \varepsilon_{*}+\delta\right),
$$

we deduce with $(5.54),(5.56)$ that $\left(v_{* *}(\varepsilon), \varepsilon\right)$ is a solution to $(2.14)$. Moreover, with

$$
\varphi(\varepsilon)=\left(\varepsilon-\varepsilon_{*}\right)^{1 / 2} \beta \varphi_{* *}+o\left(\varepsilon-\varepsilon_{*}\right)^{1 / 2},
$$

(5.67) and (5.55), we obtain

$$
v_{* *}(\varepsilon) \simeq \sqrt{\frac{2}{\tilde{\varepsilon}_{* *}(0)}\left(\varepsilon-\varepsilon_{*}\right)} \varphi_{* *} \quad \text { in } \quad \dot{V}_{0} .
$$

If we consider the solution to (5.69) satisfying $\tilde{\varphi}(0)=-\beta \varphi_{* *}$, we obtain the "negative" branch of $v_{* *}$. Hence (5.63) follows which completes the proof of the Theorem.

\section{NumericAl SimUlations}

The results of Section 5 provide a description of solutions to equation (2.14) near a bifurcation point. In this section, we illustrate and extend these results by numerical simulations. 


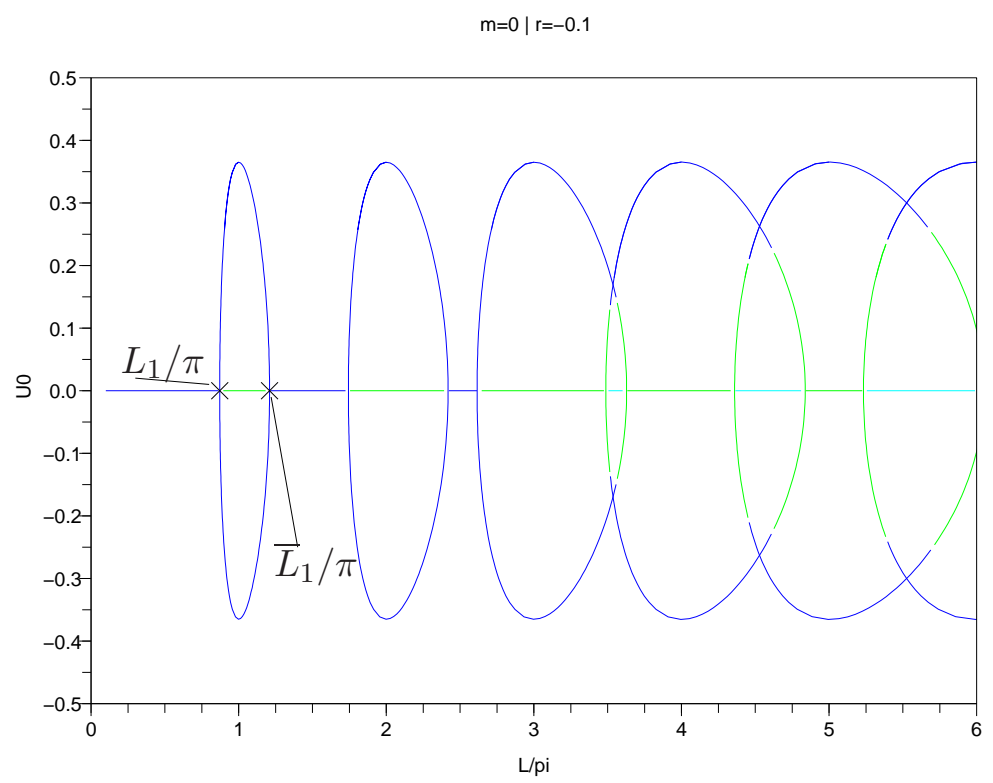

Figure 3. Case $M=0, r=-0.1(p=0.1)$

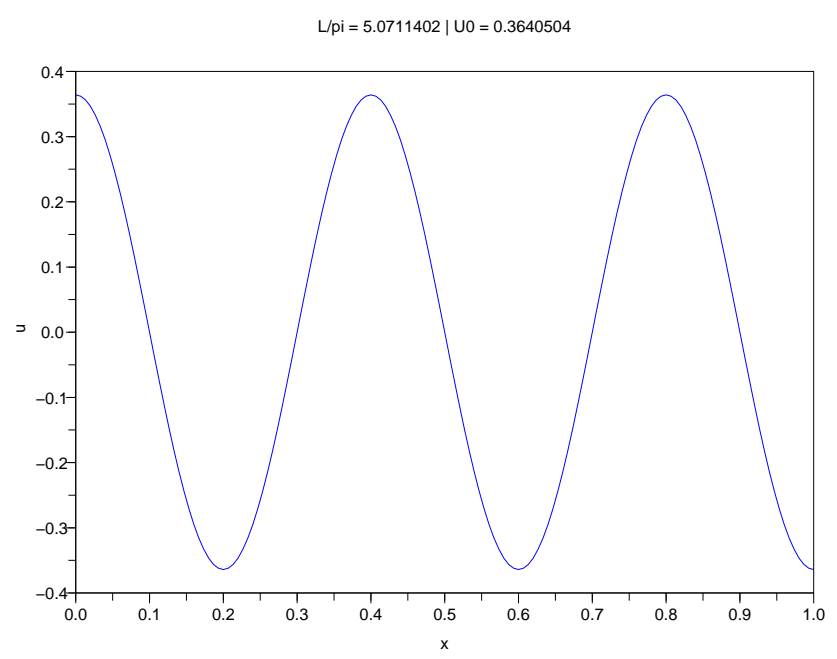

FIgURE 4. Stable solution for $M=0, r=-0.1(L \approx 5.07 \pi)$

6.1. Numerical method and technical aspects. In order to deal easily with the boundary conditions, we have chosen to discretize equation (2.14), or equivalently, (2.8), by a finite element method. In order to deal with the bilaplacian term, we use a splitting method as in, for instance, [EFM89, IP08, BRV07]. More precisely, letting $w=-\varepsilon u^{(2)}$, 
we see that $(2.8)$ is equivalent to finding $(u, w, c) \in W_{0} \times W_{0} \times \mathbb{R}$ such that

$$
\left\{\begin{array}{l}
w=-\varepsilon u^{(2)} \\
-\varepsilon w^{(2)}-2 w+f(u)=c \\
\int_{0}^{1} u \mathrm{~d} x=M .
\end{array}\right.
$$

Define $X=H^{1}(0,1)$. The variational formulation of (6.1) is: find $(u, w, c) \in X \times X \times \mathbb{R}$ such that

$$
\begin{cases}(w, \varphi)=\varepsilon\left(u^{\prime}, \varphi^{\prime}\right), & \forall \varphi \in X \\ \varepsilon\left(w^{\prime}, \chi^{\prime}\right)-2(w, \chi)+(f(u), \chi)=c(1, \chi), & \forall \chi \in X \\ (u, 1)=M, & \end{cases}
$$

where $(\cdot, \cdot)$ denotes the $L^{2}(\Omega)$-scalar product. Notice that by elliptic regularity, any solution $(u, w, c) \in X \times X \times \mathbb{R}$ of (6.2) satisfies $u, w \in H^{2}(\Omega)$; integration by parts shows that the boundary conditions are also satisfied, so that $u, w \in W_{0}$ and any solution of (6.2) is also a solution of (6.1) (the converse is obvious).

For the discretization of (6.2) we use conforming $P^{1}$ finite elements. We let $h=$ $1 /(N-1)$ with $N \geq 2$, we let $x_{i}=i h$ for $i=0,1, \ldots, N-1$ and we define

$$
X^{h}=\left\{u^{h} \in \mathcal{C}^{0}([0,1]), u_{\mid\left[x_{i}, x_{i+1}\right]}^{h} \text { is affine } \forall i \in\{0, \ldots, N-2\}\right\} .
$$

The discrete version of (6.2) reads: find $\left(u^{h}, w^{h}, c\right) \in X^{h} \times X^{h} \times \mathbb{R}$ such that

$$
\begin{cases}\varepsilon\left(u^{h \prime}, \varphi^{h \prime}\right)-\left(w^{h}, \varphi^{h}\right)=0, & \forall \varphi^{h} \in X^{h} \\ \varepsilon\left(w^{h \prime}, \chi^{h \prime}\right)-2\left(w^{h}, \chi^{h}\right)+\left(f\left(u^{h}\right), \chi^{h}\right)-c\left(1, \chi^{h}\right)=0, & \forall \chi^{h} \in X^{h} \\ \left(u^{h}, 1\right)-M=0 . & \end{cases}
$$

For the actual resolution of (6.3), we use the nodal basis $\left(\chi_{i}\right)_{0 \leq i \leq N-1} \in\left(X^{h}\right)^{N}$, defined by

$$
\chi_{i}\left(x_{j}\right)=\delta_{i j}, \quad 0 \leq i, j \leq N-1 .
$$

Letting $u^{h}=\sum_{i=0}^{N-1} u_{i} \chi_{i}, w^{h}=\sum_{i=0}^{N-1} w_{i} \chi_{i}$, and defining the matrices

$$
(A)_{i j}=\left(\chi_{i}^{\prime}, \chi_{j}^{\prime}\right), \quad(B)_{i j}=\left(\chi_{i}, \chi_{j}\right), \quad \text { for } 0 \leq i, j \leq N-1
$$

and

$$
U=\left(\begin{array}{c}
u_{0} \\
\vdots \\
u_{N-1}
\end{array}\right), \quad W=\left(\begin{array}{c}
w_{0} \\
\vdots \\
w_{N-1}
\end{array}\right), \quad F^{h}(U)=\left(\begin{array}{c}
\left(f\left(u^{h}\right), \chi_{0}\right) \\
\vdots \\
\left(f\left(u^{h}\right), \chi_{N-1}\right)
\end{array}\right)
$$

the matrix formulation of (6.3) reads

$$
\left\{\begin{array}{l}
\varepsilon A U-B W=0 \\
\varepsilon A W-2 B W+F^{h}(U)-c D=0 \\
D^{t} U-M=0
\end{array}\right.
$$

where $D \in \mathbb{R}^{N}$ is defined by $(D)_{i}=\left(\chi_{i}, 1\right)$ for $0 \leq i \leq N-1$. Denoting $G(U, W, c, \varepsilon)$ the left-hand side of (6.4), we are left to study

$$
G(U, W, c, \varepsilon)=0
$$

with $G: \mathbb{R}^{N} \times \mathbb{R}^{N} \times \mathbb{R} \times(0,+\infty) \rightarrow \mathbb{R}^{N} \times \mathbb{R}^{N} \times \mathbb{R}$ smooth. 
Equation (6.5) defines implicitly $(U, W, c)$ as a function of $\varepsilon$, in the neighbourhood of any solution $(\bar{U}, \bar{W}, \bar{c}, \bar{\varepsilon})$ such that $\partial_{U, W, c} G(\bar{U}, \bar{W}, \bar{c}, \bar{\varepsilon})$ is invertible. For the numerical resolution of (6.5), we use standard path-following methods. We have more precisely implemented, with the SCILAB software, a version of the predictor-corrector Algorithm 2.3.3 in [Mei00], with a fixed stepsize. Detection of bifurcation points and branch switching at such points were also treated by standard methods [Mei00]. In view of (5.25), the starting point $\left(U_{0}, W_{0}, c_{0}, \varepsilon_{0}\right)$ on a branch is (generally) found by setting $\varepsilon_{0}=1 / \lambda_{k}$ and by computing $\left(U_{0}, W_{0}, c_{0}\right)$ by a Newton algorithm initialized with a $\varphi_{k}=\cos (k \pi \cdot)$ profile; the amplitude of this initial guess is chosen by testing several cases.

We present here some of the bifurcation diagrams that were obtained, with $h=1 / 150$. For numerical integration, the Gauss quadrature formula of order 5 with 3 points was used, so that the nonlinear term is computed exactly (up to computer accuracy). For the ease of representation, the $x$-axis in every bifurcation diagram represents $L / \pi$, with $L=\varepsilon^{-1 / 2}$ (see Section 5 for an interpretation of $L$ ), and the $y$-axis represents $u^{h}(0)=u_{0}$. For this reason, we will equivalently consider $L$ or $\varepsilon$ as the bifurcation parameter, and we define

$$
L_{k}:=\varepsilon_{k}^{-1 / 2} \text { and } \bar{L}_{k}:=\bar{\varepsilon}_{k}^{-1 / 2} .
$$

Note that $p=1-f^{\prime}(M)=-3 M^{2}-r$.

In the figures, the blue color is associated to stable solutions; more generally, every color represents a certain number of negative eigenvalues of the hessian of the energy (2.9) at $u$ in $\dot{W}_{0}$ ( 0 is blue, 1 is green, 2 is cyan, 3 is red, 4 is purple, 5 is yellow, 6 is black). For a discrete solution $u^{h}$ of (6.3), this number was computed numerically, as explained below. It is possible to show that this number of negative eigenvalues is equal to the dimension of the unstable manifold of the solution $u$ considered as an equilibrium point of the evolution phase field crystal equation (2.7).

We first find the discrete energy associated to the formulation (6.4): eliminating $W$, we see that (6.4) is equivalent to finding $U \in \mathbb{R}^{N}$ such that

$$
\left\{\begin{array}{l}
\varepsilon^{2} A B^{-1} A U-2 \varepsilon A U+F^{h}(U)=c D, \\
D^{t} U=M .
\end{array}\right.
$$

Noticing that $F^{h}(U)=\nabla G^{h}(U)$, where

$$
G^{h}(U)=\int_{\Omega} g\left(\sum_{i=0}^{N-1} u_{i} \chi_{i}(x)\right) \mathrm{d} x, \quad \text { with } g^{\prime}(\sigma)=f(\sigma),
$$

we see that $U$ satisfies (6.7) if and only if $U$ is a critical point of the energy

$$
E^{h}(U)=\frac{\varepsilon^{2}}{2} U^{t} A B^{-1} A U-\varepsilon U^{t} A U+G^{h}(U)
$$

under the constraint $D^{t} U=M$; the constant $c$ is a Lagrange multiplier. In order to find the number of negative eigenvalues associated to $U$, we compute numerically the spectrum of the hessian of $E^{h}$ at $U$, restricted to the subspace $\left\{V \in \mathbb{R}^{N}: D^{t} V=0\right\}$.

6.2. Numerical results. The figures are organized as follows. We first have represented, in Figures 3-9, some numerical results for the value $M=0$ and various negative values of $r$, namely, $r=-0.1,-0.25,-0.5,-0.75$ and -1 . Then, in Figures 10-16, we show some results for the value $r=-0.5$ and various positive values of $M$, namely $M=0.1, M=0.24$ and $M=\sqrt{0.4 / 3} \approx 0.365$ (notice that negative values of $M$ can 


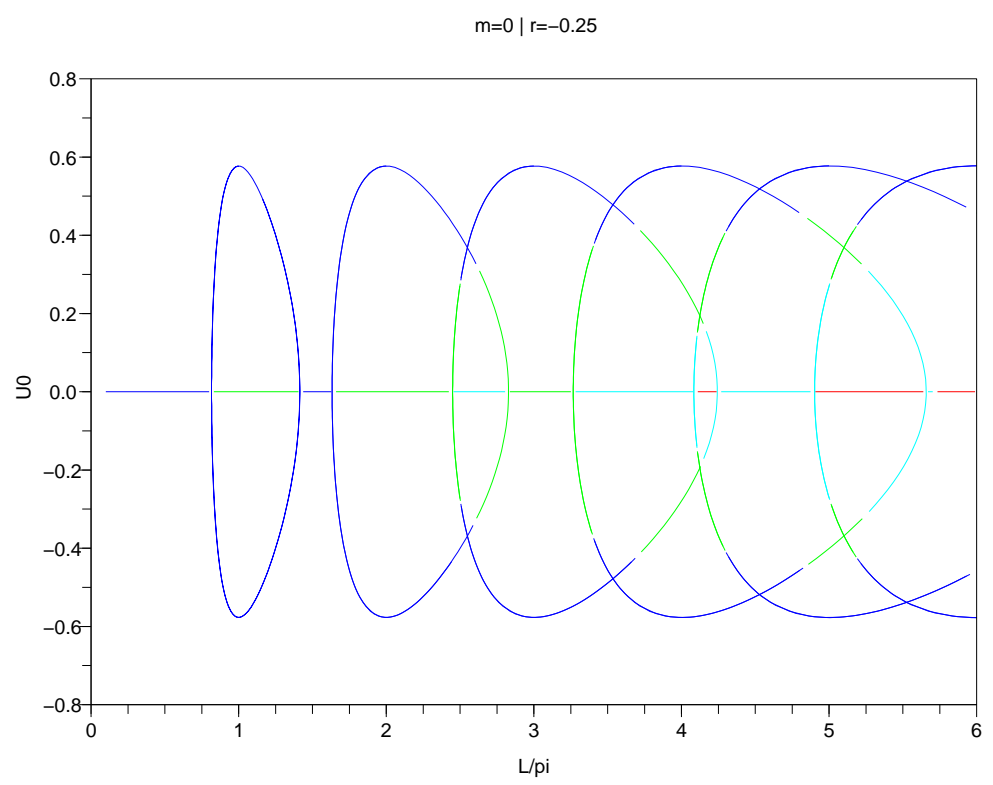

Figure 5. Case $M=0, r=-0.25(p=0.25)$

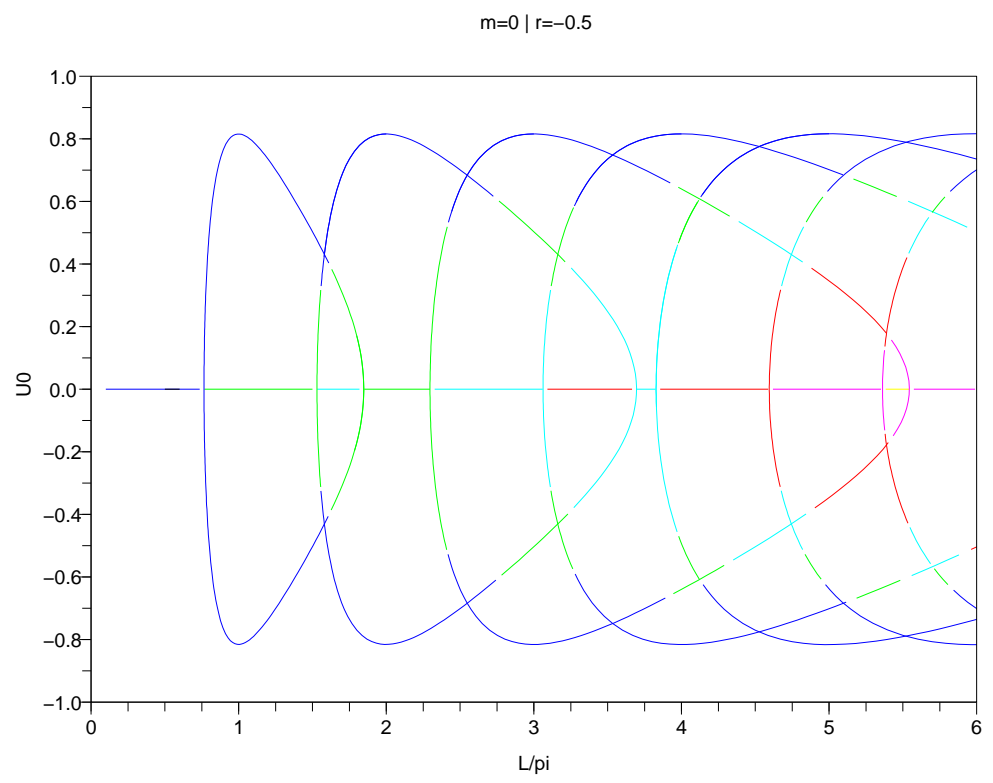

Figure 6. Case $M=0, r=-0.5(p=0.5)$

be deduced by symmetry, changing $M$ into $-M$, so that we may only consider $M \geq 0$ ). Finally, in Figures 18-22, the value of $M$ is 2 , and $r$ takes the values $-12.1,-12$ and -11.9 . 


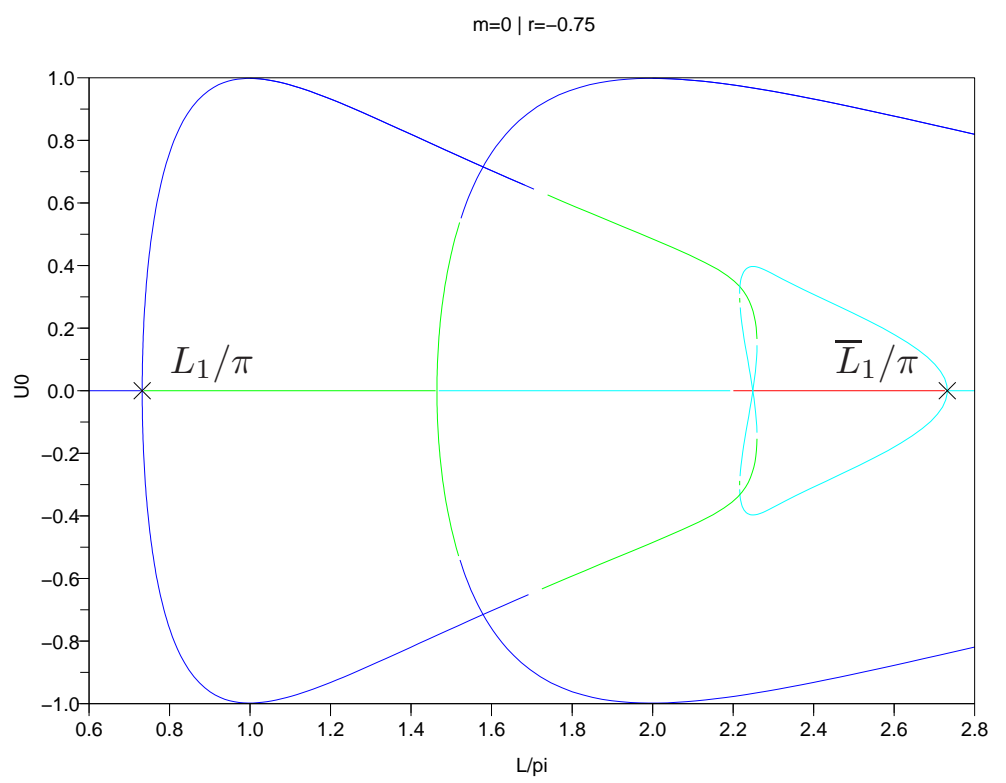

FiguRE $7.1^{\text {st }}$ and $2^{\text {nd }}$ curve for $M=0, r=-0.75(p=0.75)$

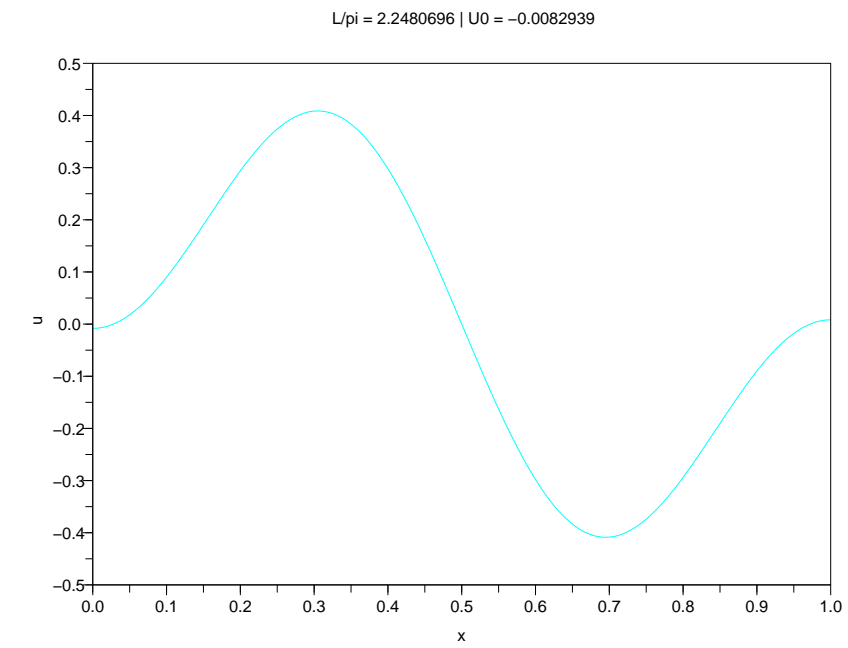

FiguRE 8. An unstable solution for $M=0, r=-0.75(L \approx 2.25 \pi)$

In Figure 3, for $M=0$ and $r=-0.1$, with $L$ varying from $0^{+}$to $6 \pi$, the horizontal line corresponds to the trivial solution $u \equiv 0$. The first curve on the left which intersects this horizontal line corresponds to a bifurcating branch initialized with a $\varphi_{1}$ profile. The intersections occur at

$$
L_{1}=\varepsilon_{1}^{-1 / 2} \approx 0.87 \pi \text { and } \bar{L}_{1}=\bar{\varepsilon}_{1}^{-1 / 2} \approx 1.21 \pi .
$$


The numerical values of $L_{1}$ and $\bar{L}_{1}$ (which can be found by a zoom on the graphic) are in excellent agreement with the theoretical values given by (4.4).

Similarly, the $k$ th curve on Figure 3 (counting from left to right, and for $k$ from 1 to 6 ) corresponds to a bifurcation branch initialized by a $\varphi_{k}$ profile: it intersects the horizontal line at $L_{k}$ and $\bar{L}_{k}>L_{k}$. The solution on this curve is $2 / k$-periodic, in agreement with Proposition 5.8 (which states this only locally, near the bifurcation). Notice that when two different curves intersect on Figure 3, it does not generally correspond to a bifurcation, but just to a common value of $u(0)$ and $L$. Figure 4 represents for instance the profile of a (numerical) solution $u$ on the 5th curve, for $L \approx 5.07 \pi$ : it is a stable solution close to (but distinct from) $u(0) \varphi_{5}$.

Recall that the blue color is associated to stable states. In Figure 3, we observe that for $L$ small ( $\varepsilon$ large), the constant is stable, and for $L$ large ( $\varepsilon$ small), it is unstable. The stability of the trivial solution is exactly as described in Proposition 4.2 , with $k_{+}=3$ and $L_{3} \approx 2.61 \pi$. The Principle of Exchange of Stability is satisfied for $\varepsilon \geq \varepsilon_{k_{+}}$, as predicted by Theorem 5.5. We also see that when $L / \pi=k$ with $k$ an integer, the stable solution has a $\varphi_{k}$-like profile: we recover here a fundamental feature of the Phase Field Crystal model for small $M$, as pointed out by Elder and Grant [EG04, Section II.B and Fig. 2]. For non integer values of $L / \pi$, there are sometimes two different profiles of stable states: for instance, when $L \approx 5.55 \pi$, the $\varphi_{5}$ and $\varphi_{6}$-like profiles are both stable.

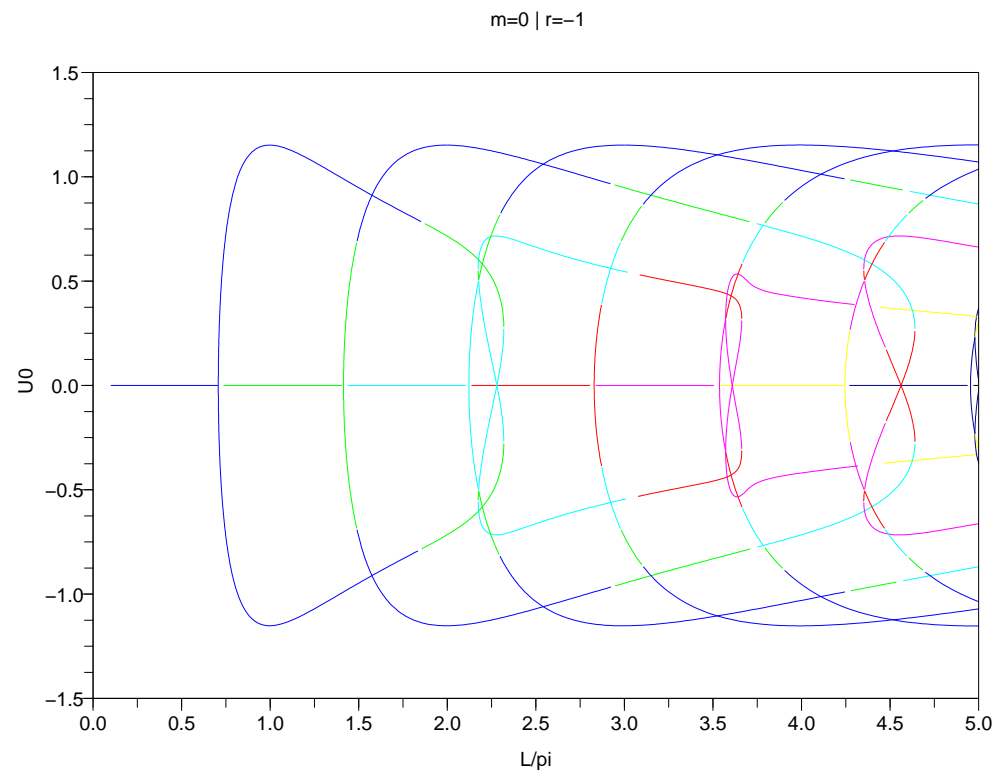

Figure 9 . Case $M=0, r=-1(p=1)$

In Figure 5, which corresponds to the case $M=0$ and $r=-0.25$, we observe similar features: for $k=1,2, \ldots$, there is a curve joining $L_{k}$ to $\bar{L}_{k}$, and which corresponds to a $\varphi_{k}$-like profile. As previously, every curve is symmetric with respect to the horizontal axis: this illustrates the fact that if $u$ is a solution of (2.8) for $M=0$, then so is $-u$; in this case, the solution $T u(\cdot+1 / k)$, given by Proposition 5.9, is equal to $-u$. The stability of the constant is again as detailed in Proposition 4.2 , with $k_{+}=2$. For $L / \pi=k$ with $k$ 
an integer, the stable state has a $\varphi_{k}$ profile. We note that every curve is the boundary of a convex domain: this agrees with Theorem 5.3 (which only proves this assertion near the bifurcation points).

Figure 6 , for $M=0$ and $r=-0.5$ displays the same features (with $k_{+}=1$ this time). Something new happens in Figure 7, for $M=0$ and $r=-0.75$, where we have only represented the first and second curves, for sake of clarity: the first curve is more complex, and it does not define the boundary of a convex domain any more. In addition to the $\varphi_{1}$ profile near $L_{1} \approx 0.73 \pi$ and $\bar{L}_{1} \approx 2.73 \pi$, a new (yet unstable) profile arises for some $L$ between these two limiting values: it is illustrated on Figure 8 for $L \approx 2.25 \pi$. A similar phenomenon happens for every curve.

Figure 9 is the case $M=0$ and $r=-1$. Since $p=1$, there is only one positive bifurcation value $\varepsilon_{k}$, for every integer $k$. Every curve starting near $L_{k}$ with a $\varphi_{k}$ profile looks like an elaborated version of the curve in Figure 8: some new (unstable) profiles appear; the curve does not seem to end for $L$ large: this agrees with the fact that $\bar{L}_{k}$ goes to $+\infty$ as $p \rightarrow 1^{-}$. Otherwise, the bifurcation diagram shows the same features (symmetry, stable solutions, local convexity) as the previous ones.

Let us point out that the bifurcation diagrams for $M=0$ and $r<-1$ (which are not shown here, but that we have computed for $r$ down to -12) show the same features as in the case $M=0, r=-1$. We also point out that the solutions $(u, w, c)$ of $(6.1)$ corresponding to the bifurcation diagrams of Figures 3-9 with $M=0$ satisfy (at least numerically) $c=0$. These solutions are therefore solution of the Swift-Hohenberg equation (compare with the results in [PR03, PR04, PW07, VdBPT01]).

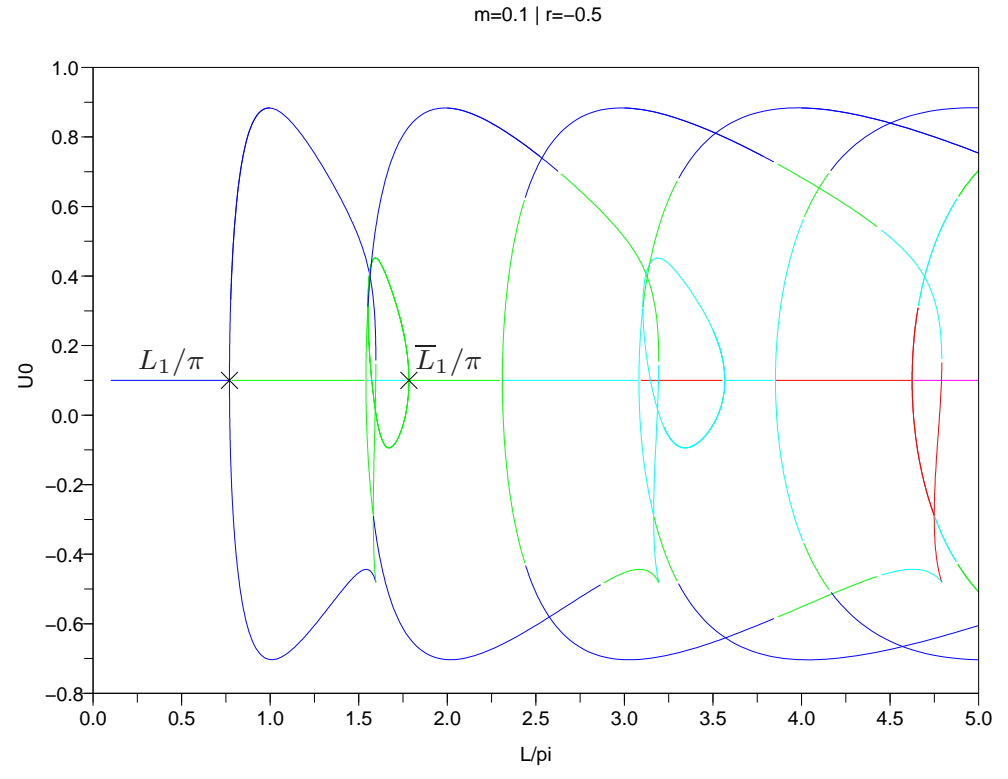

Figure 10. Case $M=0.1, r=-0.5(p=0.47)$

Figure 10 is the bifurcation diagram for $M=0.1, r=-0.5$ : it has many differences with the case $M=0, r=-0.5$ of Figure 6 . There is no longer one single curve joining $\varepsilon_{k}$ and $\bar{\varepsilon}_{k}$; there is one loop starting from $\varepsilon_{k}$, and a different (small) loop starting from $\bar{\varepsilon}_{k}$. 
$\mathrm{L} / \mathrm{pi}=1.5966707 \mid \mathrm{U} 0=-0.4808126$

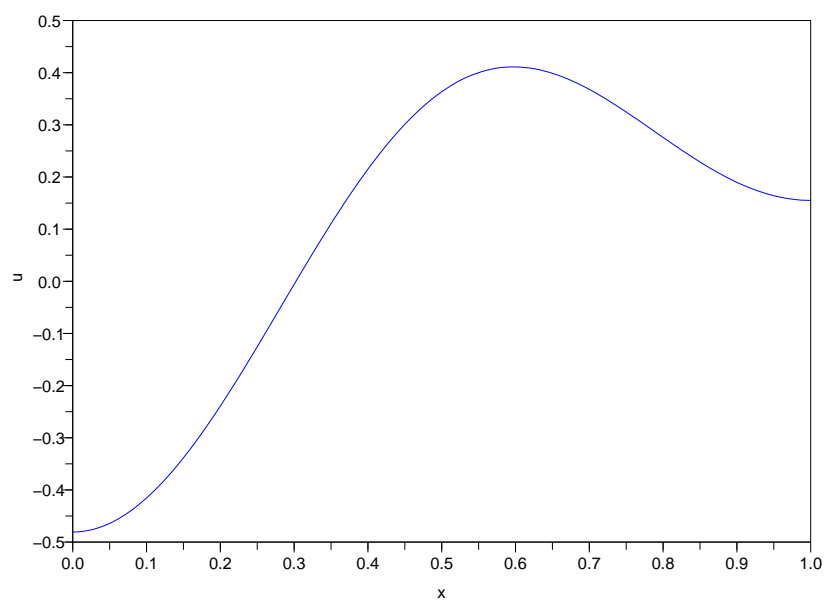

Figure 11. A stable solution $M=0.1, r=-0.5(L \approx 1.60 \pi)$

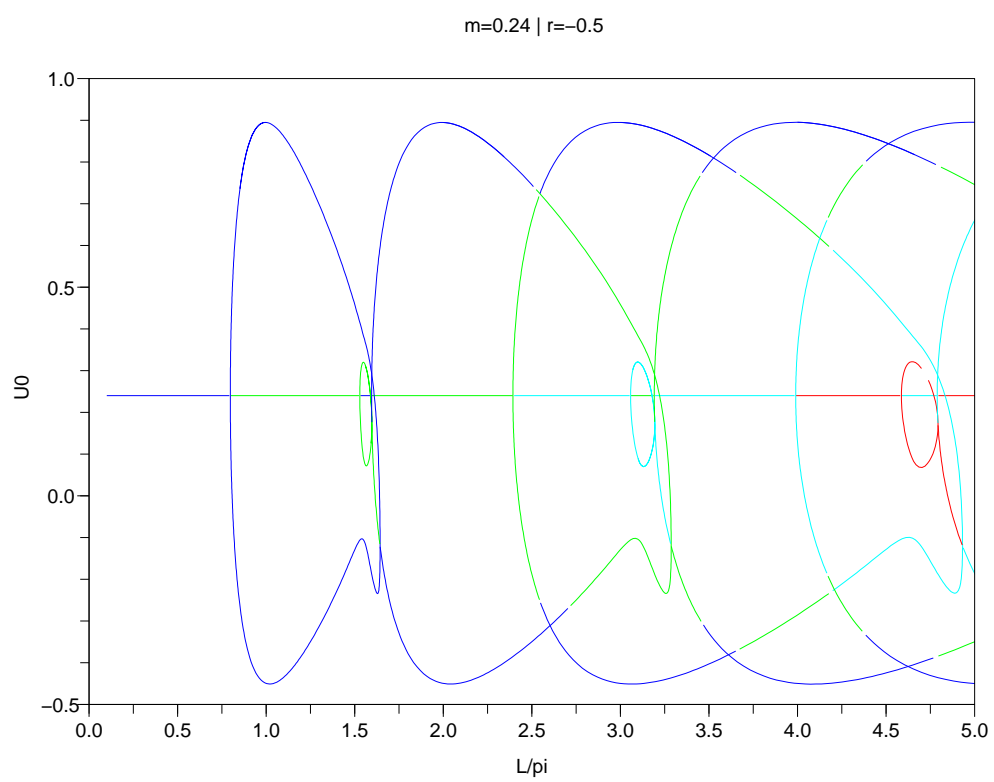

Figure 12. Case $M=0.24, r=-0.5(p=0.3272)$

These curves are no longer symmetric with respect to the horizontal axis: this illustrate the fact that equation (2.8) has less symmetry when $M \neq 0$, since there is no more reason for $2 M-u$ to be a solution of (2.8) if $u$ is. Every curve is locally convex at $\varepsilon_{k}$ or $\bar{\varepsilon}_{k}$, in agreement with Theorem 5.3 (notice that $p=0.47>9 / 25=0.36$ ). The curve associated to $\varepsilon_{k}$ is no longer the boundary of a convex domain: this reflects the fact that the profile of a solution is close to $\varphi_{k}$ for $\varepsilon$ close to $\varepsilon_{k}$, but it undergoes some important changes when $\varepsilon$ is far from $\varepsilon_{k}$. This is illustrated in Figure 11 by the profile of a stable 


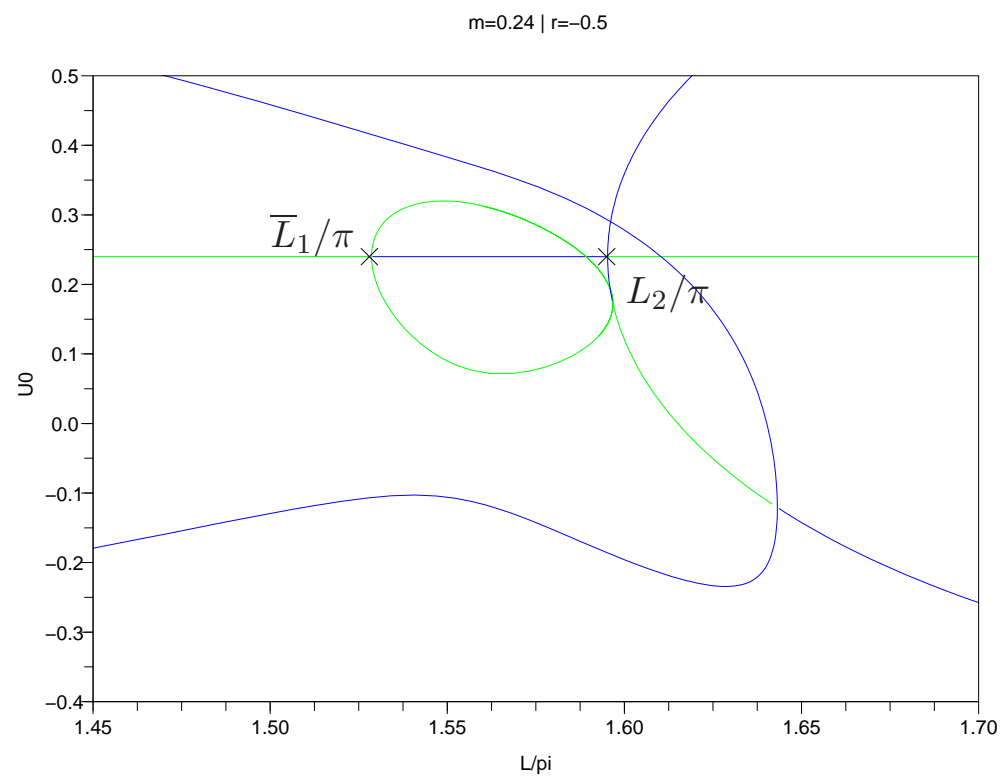

Figure 13. Case $M=0.24, r=-0.5$ (detail)

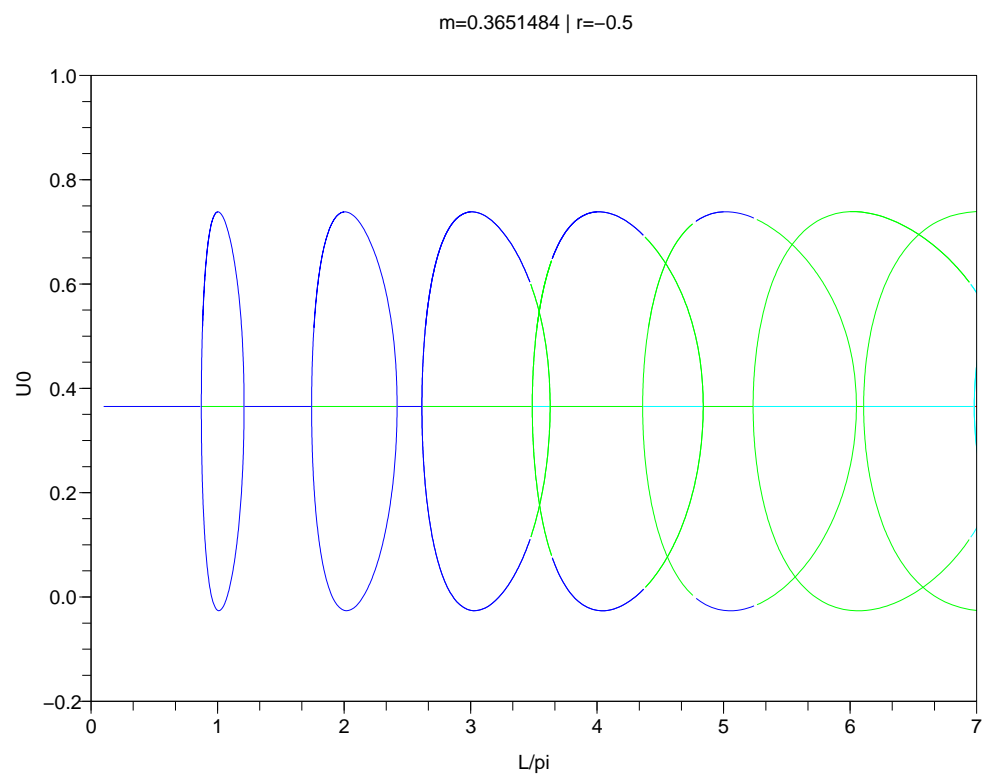

Figure 14 . Case $M=\sqrt{0.4 / 3} \approx 0.365, r=-0.5(p=0.1)$

solution for $L \approx 1.60 \pi$; this solution belongs to the curve bifurcating from $L_{1} \approx 0.77 \pi$. However, we still notice that for $L=k \pi$ with $k$ integer, the stable solution has a $\varphi_{k}$-like profile, as in the previous cases. 


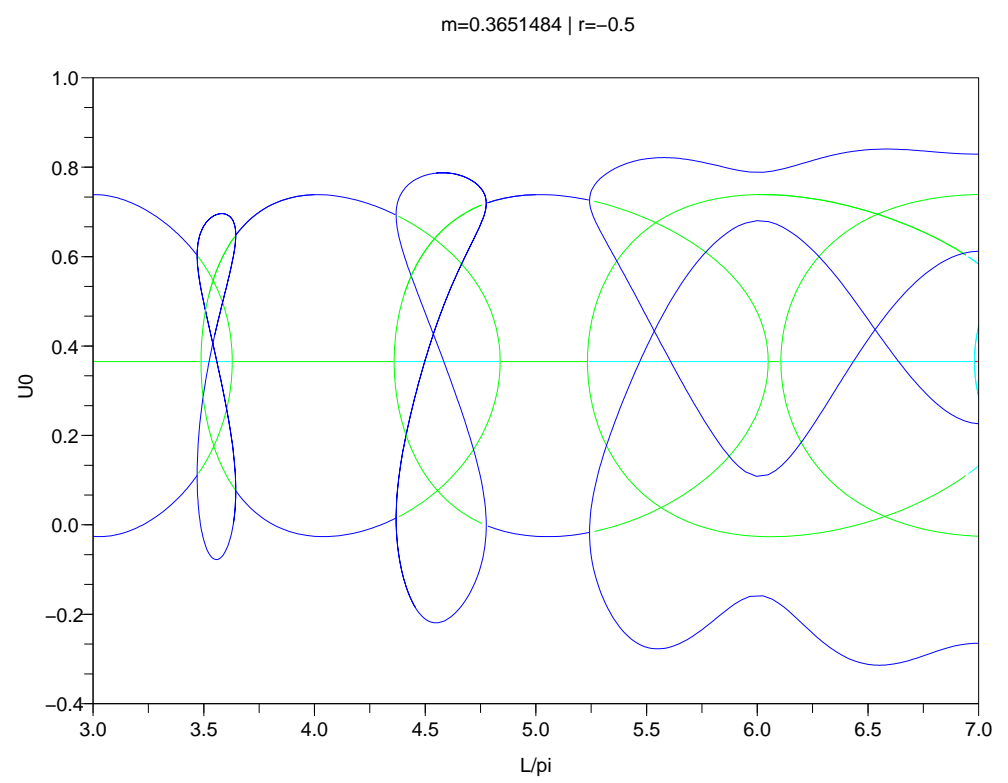

Figure 15. Case $M=\sqrt{0.4 / 3}, r=-0.5$ (detail, with the stable state)

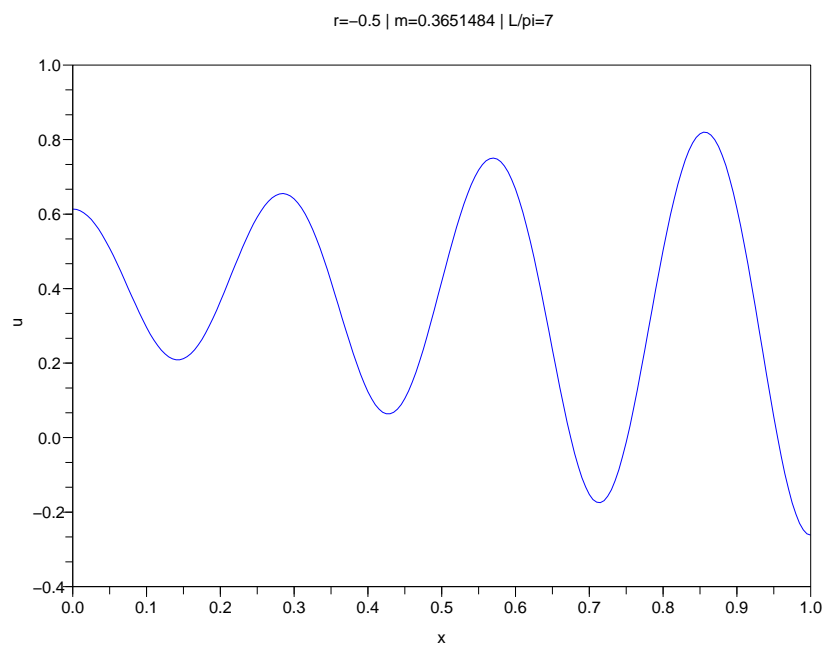

Figure 16. Solution of least energy for $M=\sqrt{0.4 / 3}, r=-0.5$ and $L=7 \pi$

Figure 12, which is the case $M=0.24$ and $r=-0.5$, is similar to Figure 10 in many ways. The main difference it that the curve associated to $\bar{\varepsilon}_{k}$ is locally concave at $\bar{\varepsilon}_{k}$ (see also Figure 13 - note that the right intersection of the small closed curve with the horizontal line is not a bifurcation point; the left intersection is $\bar{L}_{1} \approx 1.53$ ), in agreement with Theorem 5.3 and Definition 5.1. Indeed, in this case we have $M^{2}<3 / 2, p_{0} \approx 0.301$ and $p=0.3272$ so that $p_{0}<p<9 / 25$. The curve associated to $\varepsilon_{k}$ is seen to be locally convex at $\varepsilon_{k}$, as predicted since $M^{2}<3 / 2$. We also notice that the curve starting from 


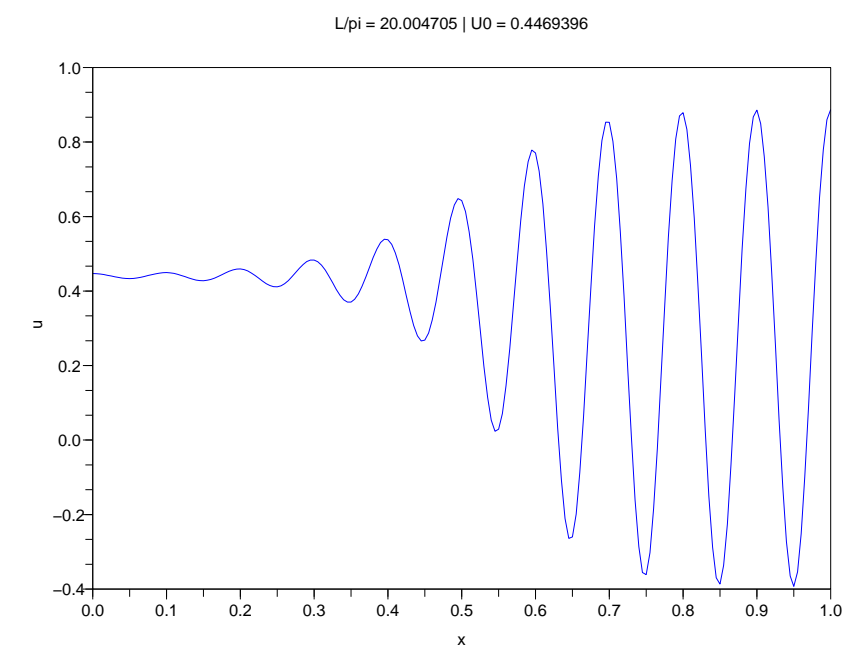

Figure 17. Solution of least energy for $M=\sqrt{0.4 / 3}, r=-0.5$ and $L \approx 20 \pi$

$\bar{\varepsilon}_{k}$ and the curve starting from $\varepsilon_{2 k}$ are tangent at $u(0) \approx 0.17$ (see Figure 13 for the case $k=1$ and $L \approx 1.6 \pi)$. For both curves, the profile of the solution at the point of tangency is (graphically) close to $M+(u(0)-M) \varphi_{2 k}$.

In Figure 14, we still have $r=-0.5$, but $M=\sqrt{0.4 / 3} \approx 0.365$ is slightly larger than previously. The bifurcation diagram is similar to that of Figure $3(M=0, r=-0.1)$ : there is only one curve joining $\varepsilon_{k}$ to $\bar{\varepsilon}_{k}$, and this curve is (surprisingly) symmetric with respect to the constant solution, and convex. The (apparent) symmetry of the bifurcation diagram is a consequence of Proposition 5.9 together with the fact that the profile of a solution on the $k$ th curve is close to $M+(u(0)-M) \varphi_{k}$. In fact, we have $p=0.1$, exactly as in Figure 3, so that the values of $\varepsilon_{k}$ and $\bar{\varepsilon}_{k}$ are the same in both cases. As a consequence, the curves on Figures 14 and 3 have similar features near $\varepsilon_{k}$ and $\bar{\varepsilon}_{k}$; there is however, a big difference concerning the stable states. When $L \approx k \pi$ with $k$ integer and large (say, $k=6,7$ ), the $\varphi_{k}$ profile is no longer stable in Figure 14: its unstable manifold has dimension 1. Such a situation was partly predicted by Elder and Grant [EG04, Fig. 2]: their analysis shows that the case $M \approx 0.365$ and $r=-0.5$ corresponds to a situation where liquid and solid can coexist.

For completeness, we have computed the stable state in this case, by programming the evolution equation as in [BRV07, IP08], and starting the evolution from a random profile: the evolution equation can be seen as a minimization algorithm. The profile of the state of least energy is represented in Figure 16 for $L=7 \pi$. Starting from similar profiles, we were able to complete the bifurcation diagram of Figure 14. The result is shown in Figure 15. We observe a symmetry breaking, related to the profile of the solution of least energy. The value of $L$ is small here, in comparison with the situation analysized by Elder and Grant [EG04], so that it is not clear whether this profile represents a liquid and solid coexistence. For this reason, we have also computed solutions for larger values of $L$, starting from this profile, and using our path-following method with a smaller space stepsize $h=1 / 200$. The result is shown in Figure 17, for $L \approx 20 \pi$ : this solution is stable, and we checked that that evolution equation initialized with a random profile converges (for a large number of time steps) to a similar profile. Thus, Figure 17 represents a 
solution of least energy, and it corresponds to a liquid/solid coexistence, as predicted by Elder and Grant.

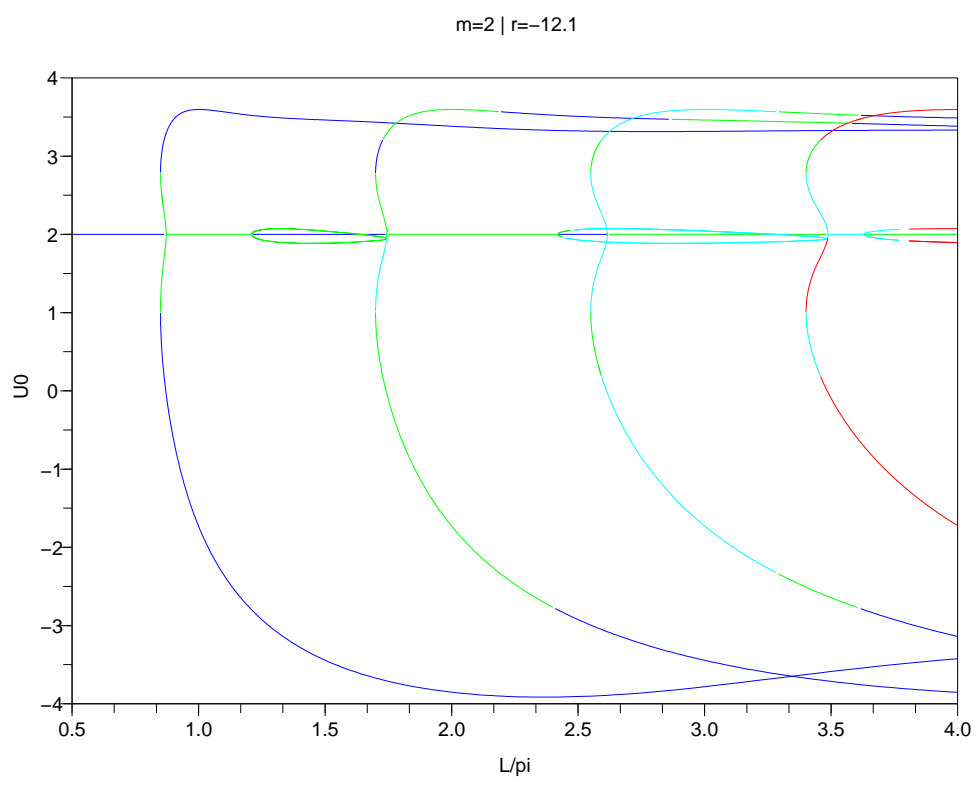

Figure 18. Case $M=2, r=-12.1(p=0.1)$

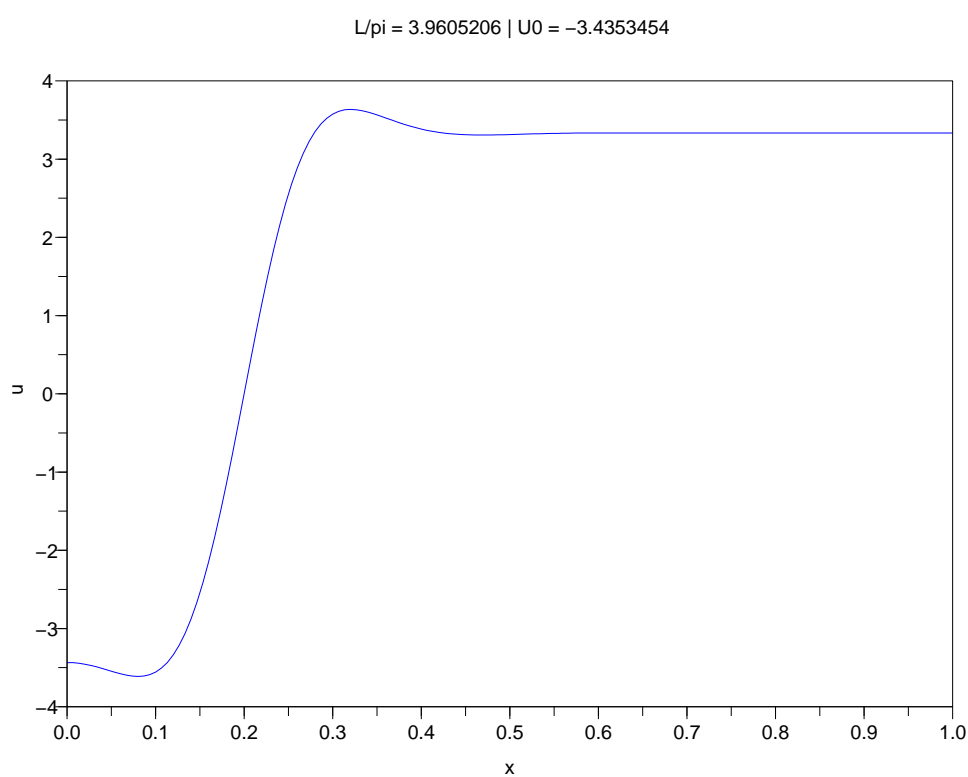

Figure 19. Solution of least energy for $M=-2, r=-12.1$ and $L / \pi \approx 3.96$ 


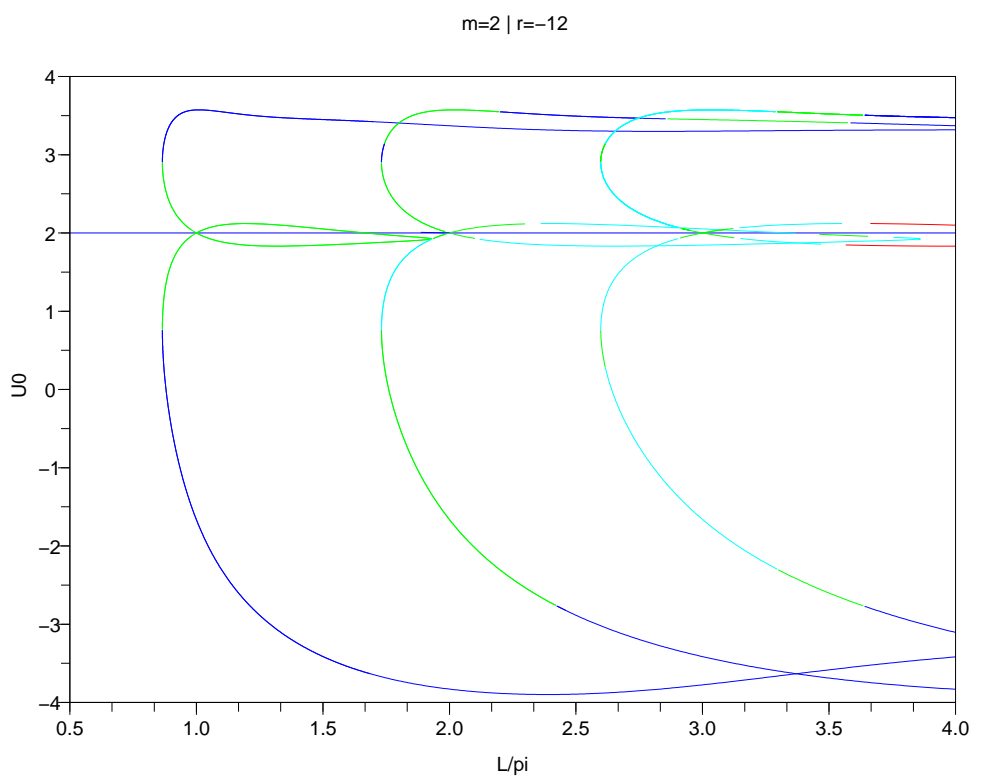

Figure 20. Case $M=2, r=-12(p=0)$

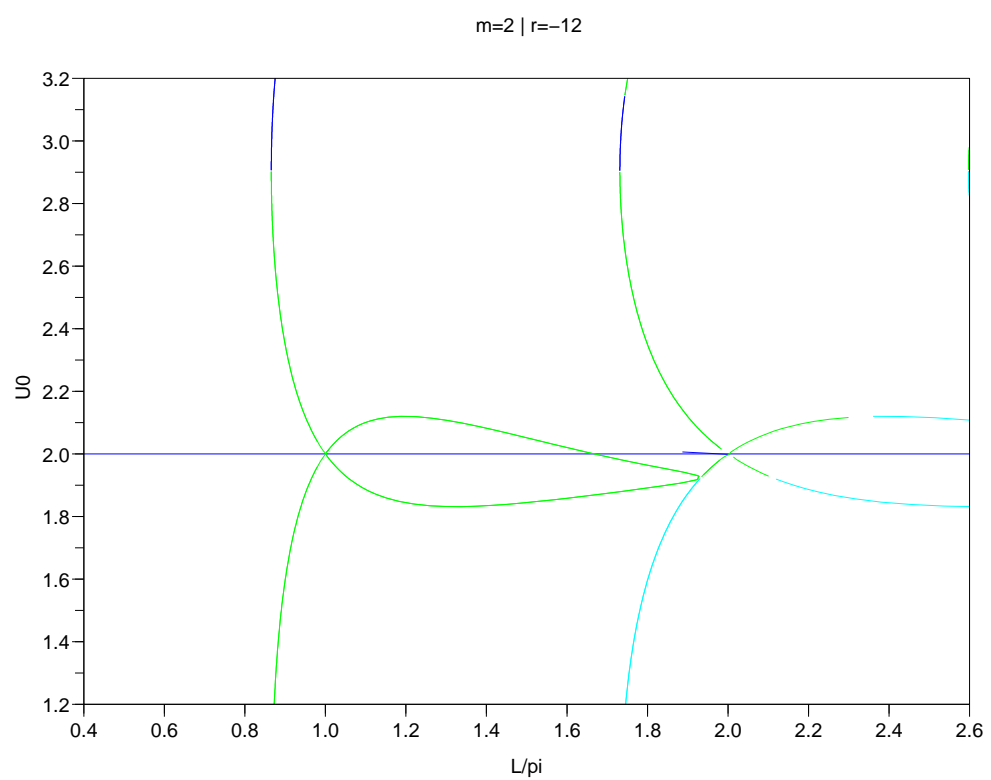

Figure 21. Case $M=2, r=-12$ (detail)

In Figure 18, the coefficients $M=2$ and $r=-12.1$ were chosen in order to illustrate a situation, predicted by Theorem 5.2, where the curve is not locally convex near $\varepsilon_{k}$. Indeed, for these coefficients, we have $M^{2}=4>3 / 2, p_{0} \approx 0.231$ and $p=0.1$, so that $p<p_{0}<9 / 25$ : the bifurcating branch is therefore concave at $\varepsilon_{k}$ and at $\bar{\varepsilon}_{k}$, as seen on 


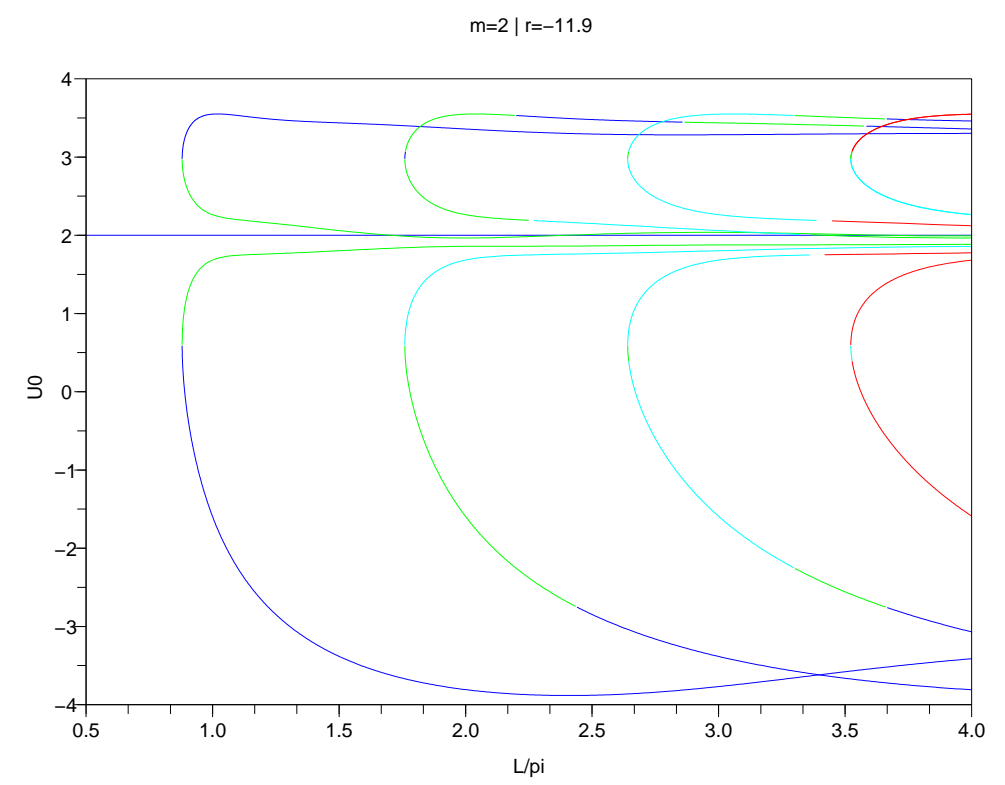

Figure 22. Case $M=2, r=-11.9(p=-0.1)$

the figure. Because of the concavity, the solution bifurcating from $\varepsilon_{1}$ is unstable near $\varepsilon_{1}$, as a consequence of the Principle of Exchange of Stability (Theorem 5.5). The solution of least energy, represented on Figure 19 for $L \approx 3.96 \pi$ has an unexpected profile, very different from $\varphi_{k}$; it was obtained by bifurcation from $\varepsilon_{1}$ with the $\varphi_{1}$ profile. This profile is also confirmed by the evolution equation started from a random profile.

Figures 20 and 21 with $M=2$ and $r=-12$ show a bifurcation diagram in the case $p=0$ : it illustrates the result of Theorem 5.7. Notice that assumption (5.37) is equivalent to $M^{2}>3 / 2$, so that it is satisfied here.

Finally, Figure 22 with $M=2$ and $r=-11.9$ shows numerical results obtained in the case $p=-0.1$. It appears as a continuous perturbation of the diagram shown in Figure 20, although our bifurcation approach is of no use here, since $p<0$ and we know that there is no bifurcation from the constant state. Other methods should be considered for a better understanding of this case.

Acknowledgements. The authors would like to thank Ken Elder for interesting discussions regarding the Phase Field Crystal model. This work was partially supported by the PEPS CPCRISTAL of the CNRS.

\section{REFERENCES}

[AP93] Antonio Ambrosetti and Giovanni Prodi. A primer of nonlinear analysis, volume 34 of Cambridge Studies in Advanced Mathematics. Cambridge University Press, Cambridge, 1993.

[BRV07] R. Backofen, A. Rätz, and A. Voigt. Nucleation and growth by a phase field crystal (pfc) model. Phil. Mag. Lett., 87(11):813-820, 2007.

[CH82] Shui Nee Chow and Jack K. Hale. Methods of bifurcation theory, volume 251 of Grundlehren der Mathematischen Wissenschaften [Fundamental Principles of Mathematical Science]. Springer-Verlag, New York, 1982. 
[EFM89] C. M. Elliott, D. A. French, and F. A. Milner. A second order splitting method for the Cahn-Hilliard equation. Numer. Math., 54(5):575-590, 1989.

[EG04] K. R. Elder and M. Grant. Modeling elastic and plastic deformations in nonequilibrium processing using phase field crystals. Phys. Rev. E, 70(051605), 2004.

$\left[\mathrm{EPB}^{+} 07\right] \quad$ K. R. Elder, N. Provatas, J. Berry, P. Stefanovic, and M. Grant. Phase-field crystal modeling and classical density functional theory of freezing. Physical Review B, 75(6), 2007.

[IP08] S. Injrou and M. Pierre. Stable discretizations of the Cahn-Hilliard-Gurtin equations. Discrete Contin. Dyn. Syst., 22(4):1065-1080, 2008.

[Kie04] Hansjörg Kielhöfer. Bifurcation theory, volume 156 of Applied Mathematical Sciences. Springer-Verlag, New York, 2004. An introduction with applications to PDEs.

[Mei00] Z. Mei. Numerical bifurcation analysis for reaction-diffusion equations, volume 28 of Springer Series in Computational Mathematics. Springer-Verlag, Berlin, 2000.

$\left[\mathrm{PDA}^{+} 07\right]$ N. Provatas, J.A. Dantzig, B. Athreya, P. Chan, P. Stefanovic, N. Goldenfeld, and K.R. Elder. Using the phase-field crystal method in the multi-scale modeling of microstructure evolution. J. of the Minerals, Metals and Materials Society, 59(7):83-90, 2007.

[PR03] L. A. Peletier and V. Rottschäfer. Large time behaviour of solutions of the Swift-Hohenberg equation. C. R. Math. Acad. Sci. Paris, 336(3):225-230, 2003.

[PR04] L. A. Peletier and V. Rottschäfer. Pattern selection of solutions of the Swift-Hohenberg equation. Phys. D, 194(1-2):95-126, 2004.

[PW07] L. A. Peletier and J. F. Williams. Some canonical bifurcations in the Swift-Hohenberg equation. SIAM J. Appl. Dyn. Syst., 6(1):208-235 (electronic), 2007.

[VdBPT01] G. J. B. Van den Berg, L. A. Peletier, and W. C. Troy. Global branches of multi-bump periodic solutions of the Swift-Hohenberg equation. Arch. Ration. Mech. Anal., 158(2):91153, 2001.

Laboratoire de Mathématiques et Applications

UMR 6086

Université DE Poitiers \& CNRS

SP2MI - BP 30179

86962 Futuroscope Chasseneuil Cedex - France

E-mail address: Morgan.Pierre@math.univ-poitiers.fr

E-mail address: Arnaud.Rougirel@math.univ-poitiers.fr 